\title{
A HIERARCHY OF MODELS FOR TURBULENT DISPERSED TWO-PHASE FLOWS DERIVED FROM A KINETIC EQUATION FOR THE JOINT PARTICLE-GAS PDF*
}

\author{
KOMLA DOMELEVO ${ }^{\dagger}$ AND PHILIPPE VILLEDIEU $\ddagger$
}

\begin{abstract}
This paper deals with the statistical modeling of turbulent two-phase flows consisting of particles or droplets immersed in a gas. The problem of gaseous turbulence alone being very complex, we concentrate here on the simpler case of an a priori given forced isotropic homogeneous turbulence acting on the particles, whose mean square velocity and integral Lagrangian time-scale are given constants. Our main objective is to derive a hierarchy of reduced models from the joint particle-gas pdf (probability density function). The latter equation may therefore be regarded as a master equation for our problem. The reduced models describe the dispersion of a cloud of particles observed at different time scales compared to the dynamic response time of the particles and the characteristic time scale of the turbulence along their trajectories. These derivations rely on very classical Chapman-Enskog expansions. We recover in particular the result of Tchen [C. M. Tchen, Mean value and correlation problems connected with the motion of small particles suspended in a turbulent fluid, PhD thesis, Delft, The Hague, Martinus Nijhoff, 1947] stating that the diffusion rate is the same for small or large particles in homogeneous turbulence, under the assumption that the lagrangian statistical properties along their paths are the same. Moreover, our approach allows us to prove that the long-time limit of the joint particle-gas distribution function is a bi-maxwellian distribution, whatever the size of the particles. This is consistent with some usual assumptions made in the literature for the derivation of particle collision models [J. Laviéville, E. Deutsch and O. Simonin, Large eddy simulation of interactions between colliding particles and a homogeneous isotropic turbulence field, Gas-Solid Flows, ASME, 228, 347-358, 1995], [Leonid I. Zaichik, Olivier Simonin and Vladimir M. Alipchenkov, Two statistical models for predicting collision rates of inertial particles in homogeneous isotropic turbulence, Phys. Fluids, 15(10), 2995-3005, 2003].
\end{abstract}

Key words. turbulence, fluid-particles interaction, hydrodynamic limits

AMS subject classifications. 35B25 35Q99

\section{Notations}

$U_{g} \quad$ Eulerian velocity field for the gas $U_{g}:=U_{g}(t, \boldsymbol{x})$

$\bar{U}_{g} \quad$ Eulerian statistical mean velocity of the gas $\bar{U}_{g}:=\bar{U}_{g}(t, \boldsymbol{x})$

$U_{g}^{\prime} \quad$ Eulerian turbulent component of the gas velocity $U_{g}^{\prime}:=U_{g}^{\prime}(t, \boldsymbol{x})$

$\boldsymbol{x}_{p}(t) \quad$ Position of a particle at time $t, \boldsymbol{x}_{p}(t):=\left(x_{p, i}(t)\right)_{i=1,2,3}$

$\boldsymbol{v}_{p}(t) \quad$ Velocity of a particle at time $t, \boldsymbol{v}_{p}(t):=\left(v_{p, i}(t)\right)_{i=1,2,3}$

$\boldsymbol{u}_{p}(t)$ Turbulent velocity of the gas along a particle's trajectory, i.e. $\boldsymbol{u}_{p}(t):=U_{g}^{\prime}\left(t, \boldsymbol{x}_{p}(t)\right)$

$\tau_{p} \quad$ Stokes repones time of the particles

$\tau_{g} \quad$ Autocorrelation turbulence time-scale along particles trajectories

$S \quad$ Stokes number, by definition $S=\tau_{p} / \tau_{g}$

$k_{g} \quad$ Turbulent kinetic energy of the gas along particles' trajectories

$\sigma_{g} \quad$ Turbulent gas mean square velocity along particles' trajectories

\footnotetext{
${ }^{*}$ Received: October 16, 2006; accepted (in revised version): March 14, 2007. Communicated by Francois Golse.

${ }^{\dagger}$ Institut de Mathématiques de Toulouse, UMR CNRS 5219, Université Paul-Sabatier (Toulouse III), 31062 Toulouse cedex 9, France (komla.domelevo@math.ups-tlse.fr).

${ }^{\ddagger}$ ONERA, 2 av. Edmond-Belin, 31055 Toulouse cedex 4, France (philippe.villedieu@onera.fr).
} 
$\mathrm{N} \quad$ Number of space dimensions

$\boldsymbol{x} \quad$ Space variable $\boldsymbol{x} \in \mathbb{R}_{\boldsymbol{x}}^{N}$

$\boldsymbol{v} \quad$ Velocity variable $\boldsymbol{v} \in \mathbb{R}_{\boldsymbol{v}}^{N}$

$\boldsymbol{u} \quad$ Turbulent gas velocity variable $\boldsymbol{u} \in \mathbb{R}_{\boldsymbol{u}}^{N}$

$\mathcal{E} \quad$ Phase space $\mathcal{E}=\mathbb{R}_{\boldsymbol{x}}^{N} \times \mathbb{R}_{\boldsymbol{v}}^{N} \times \mathbb{R}_{\boldsymbol{u}}^{N}$

$f_{\mathrm{pg}} \quad$ Joint particle-gas probability density function $f_{\mathrm{pg}}:=f_{\mathrm{pg}}(t, \boldsymbol{x}, \boldsymbol{v}, \boldsymbol{u})$

$\mathcal{M}_{1}(\boldsymbol{v} ; \sigma) \quad$ Gaussian distribution $\mathcal{M}_{1}\left(\boldsymbol{v} ; \sigma_{v}\right):=\left(1 / 2 \pi \sigma_{v}^{2}\right)^{N / 2} \exp \left(-\boldsymbol{v}^{2} / 2 \sigma_{v}^{2}\right)$

$\left.\mathcal{M}_{2}(\boldsymbol{v}, \boldsymbol{u} ; \boldsymbol{\Sigma}) \quad \mathcal{M}_{2}(\boldsymbol{v}, \boldsymbol{u} ; \boldsymbol{\Sigma})=1 /\left[(2 \pi)^{N}|\boldsymbol{\Sigma}|^{1 / 2}\right] \exp \left(-[\boldsymbol{v} ; \boldsymbol{u}]^{T} \boldsymbol{\Sigma}^{-1}[\boldsymbol{v} ; \boldsymbol{u}]\right] / 2\right)$

where $\boldsymbol{\Sigma}:=\boldsymbol{\Sigma}(\sigma, S)$ is the $2 N \times 2 N$ covariance matrix:

$$
\boldsymbol{\Sigma}:=\boldsymbol{\Sigma}\left(\sigma_{g}, S\right):=\left[\begin{array}{cc}
\mathbb{E}^{\omega} \boldsymbol{v}_{p}^{2} & \mathbb{E}^{\omega} \boldsymbol{v}_{p} \boldsymbol{u}_{p} \\
\mathbb{E}^{\omega} \boldsymbol{v}_{p} \boldsymbol{u}_{p} & \mathbb{E}^{\omega} \boldsymbol{u}_{p}^{2}
\end{array}\right]
$$

\section{Introduction}

Dispersed two-phase flows in the form of solid particles or droplets suspended in a gas are common features in many industrial and natural processes such as nuclear and combustion engineering, vulcanology, atmospheric dispersion and meteorology, amongst other areas. Almost without exception, the motion of the suspended particles is turbulent, driven by random aerodynamic forces exerted by the (high Reynolds) carrier gas flow. An important question, for all applications, is then to estimate the rate of spread of the cloud of particles in terms of the mean characteristics of the turbulence of the carrier flow. If the spreading can be modeled by a diffusion equation, then the problem reduces to finding the value of a diffusion tensor. This raises the theoretical question of deriving this diffusion equation together with the explicit expression of its coefficients, starting from the constitutive laws of motion of individual particles. This is a classical problem that has already been studied by many contributors.

Before going further in the presentation of these results, let us introduce a few notations. In the modeling of turbulent flows, it is classical to decompose the eulerian velocity field $\boldsymbol{U}_{g}(t, \boldsymbol{x})$ of the turbulent gas flow into a statistical mean component $\overline{\boldsymbol{U}}_{g}(t, \boldsymbol{x})$ and a deviation $\boldsymbol{U}_{g}^{\prime}(t, \boldsymbol{x})$, namely for all $(t, \boldsymbol{x}), \boldsymbol{U}_{g}(t, \boldsymbol{x})=\overline{\boldsymbol{U}}_{g}(t, \boldsymbol{x})+\boldsymbol{U}_{g}^{\prime}(t, \boldsymbol{x})$. Here the statistical mean should be understood as an ensemble average over all possible realisations of the gas flow $\boldsymbol{U}_{g}(\cdot, \cdot ; \omega)$ that where $\omega$ denotes a particular realisation of the flow. Hence, we have more precisely that for all $(t, \boldsymbol{x} ; \omega), \boldsymbol{U}_{g}(t, \boldsymbol{x}, \omega):=$ $\overline{\boldsymbol{U}}_{g}(t, \boldsymbol{x})+\boldsymbol{U}_{g}^{\prime}(t, \boldsymbol{x} ; \omega)$ with $\overline{\boldsymbol{U}}_{g}(t, \boldsymbol{x}):=\mathbb{E}^{\omega} \boldsymbol{U}_{g}(t, \boldsymbol{x}, \omega):=\int_{\omega} \boldsymbol{U}_{g}(t, \boldsymbol{x}, \omega) \mathrm{d} p(w)$. In the following, we will often omit the parameter $\omega$. Finally $\boldsymbol{U}_{g}^{\prime}(t, \boldsymbol{x})$ is called the turbulent velocity component of the flow.

In his pioneering work, Tchen [30] started from the basic expression of the timedependent diffusion tensor $D_{i j}(t)$ given by Taylor [29] in his theory of "diffusion by continuous movements". Here, if $\boldsymbol{v}_{p}(t)$ denote the random velocity of a particle, then for a stationary homogeneous field, the diffusion tensor $D_{i j}(t)$ writes

$$
D_{i j}(t):=\int_{0}^{t} \mathbb{E}^{\omega}\left[v_{p, i}(0) v_{p, j}(s)\right] \mathrm{d} s,
$$

where $v_{p, i}(t)$ is the $i^{t h}$ spatial component of $\boldsymbol{v}_{p}(t)$. Notice that the diffusion tensor does not depend on the initial position $\boldsymbol{x}_{p}(0)$ of the fluid particle because we assumed a stationary homogeneous turbulent velocity field. This expression applies for any type of particles, e.g. fluid particles or inertial particles. In the case of inertial particles, 
Tchen proved that the diffusion tensor (1.1) of inertial particles can be written directly in terms of the turbulence statistics provided that (i) the turbulence seen by the droplets $\boldsymbol{u}_{p}(t):=\boldsymbol{U}_{g}^{\prime}\left(t, \boldsymbol{x}_{p}(t)\right)$ is a stationary homogeneous gaussian process, (ii) the force acting on the particles is a linear drag force, and (iii) we only seek for the diffusion coefficient of the particles for large times. Namely, Tchen proved that

$$
D_{i j}(\infty):=\int_{0}^{\infty} \mathbb{E}^{\omega}\left[v_{p, i}(0) v_{p, j}(s)\right] \mathrm{d} s=\int_{0}^{\infty} \mathbb{E}^{\omega}\left[u_{p, i}(0) u_{p, j}(s)\right] \mathrm{d} s .
$$

This means that the long-time limit of $D_{i j}$ is nothing but the lagrangian autocorrelation tensor of the turbulent velocity seen along particles' trajectories. The main consequence of that result is that in a stationary homogeneous turbulent flow, the asymptotic limit of the diffusion tensor is independent of the size of the particle provided that the properties of the turbulence along the particle trajectories are size-independent. The validity of this last assumption has been widely studied in subsequent works, especially by Yudine [31] and Csanady [5] who analysed the influence of a constant external force and by Reeks [24] who also considered the effect, due to the difference between the lagrangian and eulerian integral time scales, of the turbulence on the statistics along particle paths. We come back to these difficulties in the conclusion and we refer the reader to the review paper [16] on the subject.

A more general approach based on the kinetic theory framework has been introduced by Reeks in [25] for homogeneous flows. It has been generalised to the case of nonuniform flows by several authors $[26,11,33,34]$ and fully justified in some particular cases on the basis of a rigorous mathematical derivation $[4,9]$. This fundamental approach consists of using a transport equation of Liouville type for the random instantaneous phase density function of the particles.

For that, consider an ensemble of particles characterized by their position and velocity $\left(\boldsymbol{x}_{p}(t), \boldsymbol{v}_{p}(t)\right)$ and obeying the system of ordinary differential equations

$$
\left\{\begin{array}{l}
\frac{\mathrm{d} \boldsymbol{x}_{p}}{\mathrm{~d} t}(t)=\boldsymbol{v}_{p}(t) \\
\frac{\mathrm{d} \boldsymbol{v}_{p}}{\mathrm{~d} t}(t)=\mathcal{F}\left(t, \boldsymbol{x}_{p}(t), \boldsymbol{v}_{p}(t) ; \omega\right):=\overline{\mathcal{F}}\left(t, \boldsymbol{x}_{p}(t), \boldsymbol{v}_{p}(t)\right)+\mathcal{F}^{\prime}\left(t, \boldsymbol{x}_{p}(t), \boldsymbol{v}_{p}(t) ; \omega\right),
\end{array}\right.
$$

where $\mathcal{F}^{\omega}:=\mathcal{F}(\cdot, \cdot, \cdot ; \omega)$ denotes the acceleration of a particle located at time $t$ at position $(\boldsymbol{x}, \boldsymbol{v})$ in the phase space $\mathbb{R}_{\boldsymbol{x}}^{N} \times \mathbb{R}_{\boldsymbol{v}}^{N}$ for a given realisation $\omega$ of the problem. Indeed, we assume here that the acceleration has a random component $\mathcal{F}^{\prime}\left(t, \boldsymbol{x}_{p}(t), \boldsymbol{v}_{p}(t) ; \omega\right)$. Then, the conservation of the number of particles in phase space writes in terms of its density $f_{p}^{\omega}(t, \boldsymbol{x}, \boldsymbol{v})$ as the Liouville equation

$$
\partial_{t} f_{p}^{\omega}+\nabla_{\boldsymbol{x}} \cdot\left(\boldsymbol{v} f_{p}^{\omega}\right)+\nabla_{\boldsymbol{v}} \cdot\left(\mathcal{F}^{\omega} f_{p}^{\omega}\right)=0
$$

The probabilty density function $f_{p}$ (in short "the pdf $f_{p}$ ") is by definition the statistical average of the previous random density, that is

$$
f_{p}(t, \boldsymbol{x}, \boldsymbol{v}):=\mathbb{E}^{\omega} f_{p}^{\omega}(t, \boldsymbol{x}, \boldsymbol{v}) .
$$

The pdf $f_{p}$ obviously solves

$$
\partial_{t} f_{p}+\nabla_{\boldsymbol{x}} \cdot\left(\boldsymbol{v} f_{p}\right)+\nabla_{\boldsymbol{v}} \cdot\left(\overline{\mathcal{F}} f_{p}+\mathbb{E}^{\omega}\left(\mathcal{F}^{\prime \omega} f_{p}^{\omega}\right)\right)=0,
$$

and it leads to an equation in closed form only if we can express the correlation term $\mathbb{E}^{\omega}\left(\mathcal{F}^{\prime \omega} f_{p}^{\omega}\right)$ in terms of the pdf $f_{p}$ alone. In the case of inertial particles submitted to 
a linear drag force, we have an acceleration of the form

$$
\mathcal{F}(t, \boldsymbol{x}, \boldsymbol{v} ; \omega):=\frac{1}{\tau_{p}}\left(\boldsymbol{U}_{g}(t, \boldsymbol{x} ; \omega)-\boldsymbol{v}\right):=\underbrace{\frac{1}{\tau_{p}}\left(\overline{\boldsymbol{U}}_{g}(t, \boldsymbol{x})-\boldsymbol{v}\right)}_{\overline{\mathcal{F}}(t, \boldsymbol{x}, \boldsymbol{v})}+\underbrace{\frac{1}{\tau_{p}} \boldsymbol{U}_{g}^{\prime}(t, \boldsymbol{x} ; \omega)}_{\mathcal{F}^{\prime}(t, \boldsymbol{x}, \boldsymbol{v} ; \omega)}
$$

where $\tau_{p}$ is the response time of the particles, that is the typical time needed for the velocity of the particles to relax to the velocity of the surrounding gas. It follows from (1.3) that the kinetic equation satisfied by $f_{p}$ reads

$$
\partial_{t} f_{p}+\nabla_{\boldsymbol{x}} \cdot\left(\boldsymbol{v} f_{p}\right)+\nabla_{\boldsymbol{v}} \cdot\left(\frac{\overline{\boldsymbol{U}}_{g}(t, \boldsymbol{x})-\boldsymbol{v}}{\tau_{p}} f_{p}+\mathbb{E}^{\omega}\left[\frac{\boldsymbol{U}_{g}^{\prime \omega} f_{p}^{\omega}}{\tau_{p}}\right]\right)=0 .
$$

In this pdf framework, assuming that (i) the turbulence seen by the droplets $\boldsymbol{u}_{p}(t):=\boldsymbol{U}_{g}^{\prime}\left(t, \boldsymbol{x}_{p}(t)\right)$ is a stationary homogeneous gaussian process (same hypothesis as Tchen's) and (ii) assuming a linear drag force, Reeks [25] proved that the term to estimate has the form:

$$
\mathbf{j}(t, \boldsymbol{x}, \boldsymbol{v}):=\mathbb{E}^{\omega}\left[\frac{\boldsymbol{U}_{g}^{\prime \omega} f_{p}^{\omega}}{\tau_{p}}\right]=-\left(\mathbf{A} \nabla_{\boldsymbol{v}} f_{p}+\mathbf{B} \nabla_{\boldsymbol{x}} f_{p}\right),
$$

in which

$$
\begin{aligned}
& A_{i j}(t)=\frac{1}{\tau_{p}} \mathbb{E}^{\omega}\left(u_{p, i}(t) v_{p, j}(t)\right)=\frac{1}{\tau_{p}^{2}} \int_{0}^{t} \exp \left(-\frac{t-s}{\tau_{p}}\right) R_{i j}(t, s) \mathrm{d} s, \\
& B_{i j}(t)=\frac{1}{\tau_{p}} \mathbb{E}^{\omega}\left(u_{p, i}(t) x_{p, j}(t)\right)=\frac{1}{\tau_{p}} \int_{0}^{t}\left[1-\exp \left(-\frac{t-s}{\tau_{p}}\right)\right] R_{i j}(t, s) \mathrm{d} s,
\end{aligned}
$$

and where $R_{i j}(t, s)=\mathbb{E}^{w}\left(u_{p, i}(t) u_{p, j}(s)\right)$ is the so-called lagrangian auto-correlation tensor of the turbulent velocity field (i.e. the autocorrelation tensor computed along the particles' trajectories). It is worth noticing that the expression of $R_{i j}(t, s)$ is known only if the statistics of $\boldsymbol{u}_{p}(t):=\boldsymbol{U}_{g}^{\prime}\left(t, \boldsymbol{x}_{p}(t)\right)$ can be explicitly computed. This is only possible for simple flows.

In this paper, we propose to revisit the traditional approach based on the kinetic theory by using a transport equation for the joint particle-gas probability density function $f_{\mathrm{pg}}(t, \boldsymbol{x}, \boldsymbol{v}, \boldsymbol{u})$, where the extra variable $\boldsymbol{u}$ refers to the carrier flow turbulent velocity encountered by a particle along its path. Hence by definition, the classical pdf $f_{p}:=f_{p}(t, \boldsymbol{x}, \boldsymbol{v})$ and the extended one $f_{\mathrm{pg}}:=f_{\mathrm{pg}}(t, \boldsymbol{x}, \boldsymbol{v}, \boldsymbol{u})$ are related to each other by

$$
f_{p}(t, \boldsymbol{x}, \boldsymbol{v})=\int_{\mathbb{R}_{\boldsymbol{u}}^{N}} f_{\mathrm{pg}}(t, \boldsymbol{x}, \boldsymbol{v}, \boldsymbol{u}) d \boldsymbol{u} .
$$

To obtain the equation satisfied by $f_{\mathrm{pg}}$, an extra equation is required for $\boldsymbol{u}(t)$ in addition to the particle equations of motion (please see next section). In the case of a general nonuniform fluid flow, the extra equation can be based on the model proposed by Simonin et al. [28], being a derivative of the generalized Langevin equation introduced by Pope as a stochastic lagrangian model of Navier-Stokes equations $[19,20,21]$. The main interest of this new approach is that all the turbulence 
statistics along particle trajectories have not to be prescribed a priori but are now implicitly contained in the set of the particle equations of motion.

In the present work, we derive a hierarchy of reduced models starting from the joint particle-gas pdf equation which may be regarded as a master equation for our problem. Each of these models describes the dispersion of the cloud of particles observed at a given time scale $T$ that has to be compared to the particle dynamic response time $\tau_{p}$ and to the characteristic time scale $\tau_{g}$ of the turbulence. In each case, the derivation relies on a classical Chapman-Enskog expansion which allows to eliminate one of the variables of the problem depending on which asymptotic is considered. Under suitable hypotheses, we recover the main results of Tchen's theory and also some results of Reeks, namely the expression of the diffusion tensors $A_{i j}$ and $B_{i j}$ in the relevant cases. As a by-product of this approach, we also obtain that in a uniform homogeneous isotropic fluid flow (under our modelling assumptions) the longtime limit of the joint pdf is bi-maxwellian whatever the size of the particles. This is consistent with some hypotheses made in the literature for the derivation of particle collision models $[15,32]$. The extension of this work to more general situations (simple shear flow, presence of an external force, nonlinear drag model) will be addressed in a forthcoming paper.

The paper is organized as follows. Section $\mathbf{2}$ is devoted to the derivation of the master equation for the joint particle-gas pdf, the presentation of the main results (written in dimensional variables) and finally the derivation of the nondimensional form of the problem. Then, sections $\mathbf{3}, \mathbf{4}$ and $\mathbf{5}$ are devoted to the derivations (in nondimensional variables) of the reduced models in the case of large, small or intermediate size particles respectively. We conclude the paper with an appendix where the equilibrium distributions for the bi-maxwellian are investigated thanks to entropy estimates.

\section{Modeling and main results}

2.1. Modeling assumptions and equations of motion of a single droplet.

In this work, we shall assume for the sake of simplicity the following hypotheses on the gas velocity field:

(i) The statistical mean gas velocity $\overline{\boldsymbol{U}}_{g}$ is a constant vector field. This is an important assumption because it ensures that the turbulent velocity of the gas along the particles' trajectories is not correlated the mean velocity or mean gradients, contrast for example to shear flows (see e.g. [27]). But, thanks to galilean invariance, we can assume without loss of generality that the mean velocity $\overline{\boldsymbol{U}}_{g}$ of the gas is equal to $\mathbf{0}$.

(ii) The turbulence velicity field $\boldsymbol{U}_{g}^{\prime}(t, \boldsymbol{x})$ are stationary, isotropic and homogeneous. Therefore, the statistical properties of the stochastic process $\boldsymbol{u}_{p}(t)=$ $\boldsymbol{U}_{g}^{\prime}\left(t, \boldsymbol{x}_{p}(t)\right)$ (corresponding to the fluid velocity along a given particle path) is stationary, isotropic and do not depend on the initial particle position. Finally, the turbulent kinetic energy $k_{g}$ and the autocorrelation time-scale $\tau_{g}$ of the turbulence (both along particles trajectories) write as:

$$
k_{g}:=\mathbb{E}^{\omega}\left[\boldsymbol{u}_{p}^{2}(t) / 2\right], \quad \tau_{g}:=\frac{1}{2 k_{g}} \int_{0}^{\infty} \mathbb{E}^{\omega}\left[\boldsymbol{u}_{p}(t) \boldsymbol{u}_{p}(0)\right] \mathrm{d} t
$$

Under these hypotheses, the equations of motion for a given droplet may be written as

$$
\frac{\mathrm{d} \boldsymbol{x}_{p}}{\mathrm{~d} t}(t)=\boldsymbol{v}_{p}(t), \quad \frac{\mathrm{d} \boldsymbol{v}_{p}}{\mathrm{~d} t}(t)=\frac{\boldsymbol{u}_{p}(t)-\boldsymbol{v}_{p}(t)}{\tau_{p}}
$$


where $\boldsymbol{u}_{p}(t)$ is as usual the turbulent gas velocity seen by the droplet and $\tau_{p}$ stands for the particle response time. Now, in the case of homogeneous isotropic turbulence, by analogy with the work of Pope et al. [19, 20] on lagrangian turbulence modeling, Pozorsky, Minier and Simonin [17, 23, 18] have proposed to use the following stochastic differential equation in order to describe the fluctuating gas velocity $\boldsymbol{u}_{p}(t)=\boldsymbol{U}_{g}^{\prime}\left(t, \boldsymbol{x}_{p}(t)\right)$ seen by a droplet, when observed at time scales falling in the inertial range of the fluid turbulence:

$$
\mathrm{d} \boldsymbol{u}_{p}(t)=-\frac{\boldsymbol{u}_{p}(t)}{\tau_{g}} \mathrm{~d} t+\sqrt{\frac{2 \sigma_{g}^{2}}{\tau_{g}}} \mathrm{~d} \boldsymbol{W}_{t} .
$$

This is a so-called Ornstein-Uhlenbeck process where $\boldsymbol{W}_{t}$ is the Wiener process on $\mathbb{R}^{N}$ (with $\left.\mathbb{E}^{\omega}\left(\mathrm{d} W_{t, i} \mathrm{~d} W_{t, j}\right)=\delta_{i j} \mathrm{~d} t\right)$, where $\sigma_{g}=\sqrt{2 k_{g} / N}$ is the turbulent mean square velocity, and where $\tau_{g}$ stands for the autocorrelation time of the turbulence along the particle path. Note that the probability distribution $\phi(t, \boldsymbol{u})$ of the values of $\boldsymbol{u}_{p}(t)$ tends to a gaussian distribution only after a typical time greater than $\tau_{g}$. If $\phi(0, \boldsymbol{u})$ is gaussian at time $t=0$, then it remains gaussian for all subsequent times, with a variance that tends to $\sigma_{g}^{2}$.

In order to obtain a model representing the dynamics of the whole cloud of particles, it is necessary to adopt a statistical point of view and to introduce the one-particle joint particle-gas density function $f_{\mathrm{pg}}$, representing the density of probability for a particle to be at time $t$ at position $\boldsymbol{x}$, with velocity $\boldsymbol{v}$ and seeing a fluctuating gas velocity equal to $\boldsymbol{u}$. The corresponding phase space is therefore $\mathbb{E}=\mathbb{R}_{\boldsymbol{x}}^{N} \times \mathbb{R}_{\boldsymbol{v}}^{N} \times \mathbb{R}_{\boldsymbol{u}}^{N}$. Using the Feynman-Kac representation theorem (see [14]) it follows from system (2.1)-(2.2) that $f_{\text {pg }}$ must satisfy the following deterministic Fokker-Planck equation:

$$
\partial_{t} f_{\mathrm{pg}}+\operatorname{div}_{\boldsymbol{x}}\left(\boldsymbol{v} f_{\mathrm{pg}}\right)+\operatorname{div} \boldsymbol{v}\left(\frac{\boldsymbol{u}-\boldsymbol{v}}{\tau_{p}} f_{\mathrm{pg}}\right)-\operatorname{div}_{\boldsymbol{u}}\left(\frac{\boldsymbol{u}}{\tau_{g}} f_{\mathrm{pg}}\right)-\frac{\sigma_{g}^{2}}{\tau_{g}} \Delta_{\boldsymbol{u}} f_{\mathrm{pg}}=0
$$

2.2. Main results. Starting from the master-equation $(2.3)$ for $f_{\mathrm{pg}}$, if $T$ denotes the typical time at which the system is observed, then we derive formally a series of reduced models corresponding respectively to the following scalings:

\begin{tabular}{|c|c|c|}
\hline & intermediate time-scale & macroscopic time-scale \\
\hline \multirow{2}{*}{ large particles } & $\tau_{g} \ll \tau_{p} \sim T, f_{\mathrm{pg}} \mapsto f_{p}$ & $\tau_{g} \ll \tau_{p} \ll T, f_{p} \mapsto n$ \\
& Eqs. $(2.4)-(2.5)$ & Eqs. $(2.8)-(2.9)$ \\
\hline intermediate-size & & $\max \left(\tau_{p}, \tau_{g}\right) \ll T, f_{\mathrm{pg}} \mapsto n$ \\
particles & & Eqs. $(2.8)-(2.10)$ \\
\hline \multirow{2}{*}{ small particles } & $\tau_{p} \ll \tau_{g} \sim T, f_{\mathrm{pg}} \mapsto f_{g}$ & $\tau_{p} \ll \tau_{g} \ll T, f_{g} \mapsto n$ \\
& Eqs. $(2.6)-(2.7)$ & Eqs. $(2.8)-(2.11)$ \\
\hline
\end{tabular}

These different scalings are defined thanks to the following considerations: on the one hand, the response time $\tau_{p}$ of a particle is a decreasing function of the size of the particle which tends to 0 for infinitely small particles and to infinity for infinitely large particles. Hence, for given characteristics of the turbulent flow, in particular for a given lagrangian autocorrelation time $\tau_{g}$ of the turbulence, we denote by "small particles" the case where $\tau_{p} \ll \tau_{g}$, "large particles" the case where $\tau_{p} \gg \tau_{g}$, and "intermediate-size particles" the remaining case $\tau_{p} \sim \tau_{g}$. On the other hand, we call "intermediate time-scale" the case where the observation time $T$ is chosen to be $T \sim \max \left(\tau_{p}, \tau_{g}\right)$ and we call "macroscopic time-scale" the case where the observation time $T$ is chosen to be $T \gg \max \left(\tau_{p}, \tau_{g}\right)$. Finally, the notation " $f \mapsto g$ " in the table 
above means that in the asymptotic regime considered, the original unknown $f$ of the initial problem is replaced by the unknown $g$, solution to the asymptotic model.

We show that these asymptotic limits can be derived formally by using a Chapman-Enskog expansion starting with the above-mentioned master-equation (2.3) for the joint particle-gas pdf $f_{\mathrm{pg}}$. Such derivations can be found in classical textbooks $[2,3]$ for example. We also want to mention here reference [1] where these techniques are used to derive a hierarchy of models in the context of semiconductors as well as reference [6] which deals with a similar Fokker-Planck equation similar to Eq. (2.3) above and also provides a nice introduction to the algebra of the Chapman-Enskog expansion.

Coming back to our problem, the first three limiting problems obtained starting from (2.3) respectively allow us to rewrite the joint particle-gas pdf $f_{\mathrm{pg}}:=f_{\mathrm{pg}}(t, \boldsymbol{x}, \boldsymbol{v}, \boldsymbol{u})$ in terms of $(i)$ a gaussian distribution involving the variables that vary rapidly at the scale of the observation time-scale $T$, multiplied by $(i i)$ a contribution involving the reduced distribution $f_{p}:=f_{p}(t, \boldsymbol{x}, \boldsymbol{v})$, or $f_{g}:=f_{g}(t, \boldsymbol{x}, \boldsymbol{u})$, or $n=n(t, \boldsymbol{x})$ depending on which of the three cases we consider. These new functions obey kinetic (2.5), (2.7) or macroscopic (2.8) diffusion equations. The two probability density functions $f_{p}$ and $f_{g}$ obtained at intermediate time-scale, are themselves asymptotically close, when observed at macroscopic time-scales, to distributions involving the macroscopic density number $n(t, \boldsymbol{x})$ of particles in physical space. The three manners of deriving an equation for the macroscopic density $n$ all lead to the same diffusion equation (2.8) as predicted by Tchen [30]. Finally, these equations are valid only after a shorttime boundary-layer. A rigorous mathematical analysis would show that the relevant initial conditions for the solutions to the reduced models are projections onto suitable equilibrium distributions, but this is beyond the scope of the present paper. These results are summarized below.

$$
\begin{aligned}
& \hline \hline \text { intermediate time-scale, i.e. } T \sim \max \left(\tau_{p}, \tau_{g}\right) \\
& \hline \hline f_{\mathrm{pg}}(t, \boldsymbol{x}, \boldsymbol{v}, \boldsymbol{u})=\mathcal{M}_{1}\left(\boldsymbol{u} ; \sigma_{g}\right)\left(f_{p}(t, \boldsymbol{x}, \boldsymbol{v})+\frac{\tau_{g}}{\tau_{p}} \boldsymbol{u} \cdot \nabla_{\boldsymbol{v}} f_{p}(t, \boldsymbol{x}, \boldsymbol{v})\right)+o\left(\frac{\tau_{g}}{\tau_{p}}\right)
\end{aligned}
$$

with $f_{p}$ the solution of

$$
\frac{\partial f_{p}}{\partial t}+\operatorname{div}_{\boldsymbol{x}}\left(f_{p} \boldsymbol{v}\right)+\operatorname{div}_{\boldsymbol{v}}\left(-\frac{1}{\tau_{p}} f_{p} \boldsymbol{v}-\frac{\sigma_{g}^{2} \tau_{g}}{\tau_{p}^{2}} \nabla_{\boldsymbol{v}} f_{p}\right)=0
$$

\begin{tabular}{|l|c|}
\hline \hline intermediate time-scale, i.e. $T \sim \max \left(\tau_{p}, \tau_{g}\right)$ & small particles, i.e. $\tau_{p} \ll \tau_{g}$ \\
\hline \hline
\end{tabular}

$$
f_{\mathrm{pg}}(t, \boldsymbol{x}, \boldsymbol{v}, \boldsymbol{u})=\mathcal{M}_{1}\left(\boldsymbol{v}-\boldsymbol{u} ; \sigma_{g} \sqrt{\tau_{p} / \tau_{g}}\right)\left(f_{g}(t, \boldsymbol{x}, \boldsymbol{u})\right)+O\left(\tau_{p} / \tau_{g}\right)
$$

with $f_{g}$ the solution of

$$
\frac{\partial f_{g}}{\partial t}+\operatorname{div}_{\boldsymbol{x}}\left(f_{g} \boldsymbol{u}\right)+\operatorname{div}_{\boldsymbol{u}}\left(-\frac{1}{\tau_{g}} f_{g} \boldsymbol{u}-\frac{\sigma_{g}^{2}}{\tau_{g}} \nabla_{\boldsymbol{u}} f_{g}\right)=0
$$

macroscopic time-scale, i.e. $T \gg \max \left(\tau_{p}, \tau_{g}\right)$

$$
\frac{\partial n}{\partial t}-\sigma_{g}^{2} \tau_{g} \Delta_{\boldsymbol{x}} n=0
$$


large particles, i.e. $\tau_{p} \gg \tau_{g}$

$$
f_{p}(t, \boldsymbol{x}, \boldsymbol{v})=\mathcal{M}_{1}\left(\boldsymbol{v} ; \sigma_{g} \sqrt{\tau_{p} / \tau_{g}}\right) \quad\left(n(t, \boldsymbol{x})-\tau_{p} \boldsymbol{v} \cdot \nabla_{\boldsymbol{x}} n(t, \boldsymbol{x})\right)+o\left(\tau_{p} / T\right)
$$

intermediate-size particles, i.e. $\tau_{p} \sim \tau_{g}$

$$
\begin{aligned}
f_{\mathrm{pg}}(t, \boldsymbol{x}, \boldsymbol{v}, \boldsymbol{u})=\mathcal{M}_{2}\left(\boldsymbol{u}, \boldsymbol{v} ; \sigma_{g}, \tau_{p} / \tau_{g}\right) & \left(n(t, \boldsymbol{x})-\tau_{g}\left(\frac{\boldsymbol{u}}{\tau_{p} / \tau_{g}}+\left(2+\tau_{g} / \tau_{p}\right) \boldsymbol{v}\right) \cdot \nabla_{\boldsymbol{x}} n(t, \boldsymbol{x})\right) \\
& +o\left(\max \left(\tau_{g}, \tau_{p}\right) / T\right)
\end{aligned}
$$

small particles, i.e. $\tau_{p} \ll \tau_{g}$

$$
f_{g}(t, \boldsymbol{x}, \boldsymbol{u})=\mathcal{M}_{1}\left(\boldsymbol{u} ; \sigma_{g}\right) \quad\left(n(t, \boldsymbol{x})-\tau_{g} \boldsymbol{u} \cdot \nabla_{\boldsymbol{x}} n(t, \boldsymbol{x})\right)+o\left(\tau_{g} / T\right)
$$

where $\mathcal{M}_{1}$ (resp. $\mathcal{M}_{2}$ ) denotes a suitable centered gaussian (resp. bi-gaussian) distribution, whose precise definition is given later.

2.3. Dimensionless pdf equation for a cloud of particles. Let us introduce now a macroscopic length scale $L$, a velocity scale $U$, the corresponding time-scale $T=L / U$, and the nondimensional parameters:

$$
K_{p}=\frac{\tau_{p}}{T}, K_{g}=\frac{\tau_{g}}{T}, K=\max \left(K_{p}, K_{g}\right), S=\frac{\tau_{p}}{\tau_{g}} .
$$

The Stokes number $S$ indicates whether we are in the presence of small or large particles. By definition, we call "small particles" those for which $\tau_{p} \ll \tau_{g}$, or equivalently $S \ll 1$. In this limiting case, the particles tend to behave like fluid particles. Conversely, "large particles" are by definition those for which $\tau_{p} \gg \tau_{g}$, or equivalently $S \gg 1$, that is, nearly ballistic particles. In this work, our aim is to study the dynamics of the joint particle-gas pdf $f_{\mathrm{pg}}$ observed at intermediate or large time-scale $T$, for either small or large particles, and to derive reduced models (i.e. involving fewer variables than the kinetic equation (2.3) for the joint particle-gas pdf). For that, we use asymptotic analysis involving large or small non-dimensional parameters.

Indeed, the macroscopic scales introduced above lead to the following definition for the non-dimensional rescaled variables

$$
\tilde{t}=\frac{t}{T}, \quad \widetilde{\boldsymbol{x}}=\frac{\boldsymbol{x}}{L}, \quad \tilde{\boldsymbol{v}}=\frac{\boldsymbol{v}}{U}, \quad \widetilde{\boldsymbol{u}}=\frac{\boldsymbol{u}}{U}, \quad \tilde{\sigma}_{g}=\frac{\sigma_{g}}{U}, \quad \tilde{\boldsymbol{U}}_{g}=\frac{\boldsymbol{U}_{g}}{U}
$$

and for the corresponding joint density function

$$
\tilde{f}_{\mathrm{pg}}(\tilde{t}, \widetilde{\boldsymbol{x}}, \widetilde{\boldsymbol{v}}, \widetilde{\boldsymbol{u}})=L^{N} U^{2 N} f_{\mathrm{pg}}(T \tilde{t}, L \widetilde{\boldsymbol{x}}, U \widetilde{\boldsymbol{v}}, U \widetilde{\boldsymbol{u}})
$$

We note that, by definition of $K$ and $S$, one has:

$$
K_{p}=\frac{K}{\max (1,1 / S)}=\frac{S K}{\max (1, S)}, \quad \text { and } \quad K_{g}=\frac{K}{\max (1, S)} .
$$


From this, it follows that the dimensionless joint particle-gas pdf equation writes:

$$
\begin{aligned}
\partial_{\tilde{t}} \tilde{f}_{\mathrm{pg}}+\operatorname{div}_{\widetilde{\boldsymbol{x}}}\left(\tilde{f}_{\mathrm{pg}} \widetilde{\boldsymbol{v}}\right)+ & \frac{\max (1, S)}{S K} \quad \operatorname{div} \widetilde{\boldsymbol{v}}\left[(\widetilde{\boldsymbol{u}}-\widetilde{\boldsymbol{v}}) \tilde{f}_{\mathrm{pg}}\right] \\
& -\frac{\max (1, S)}{K} \operatorname{div} \widetilde{\boldsymbol{u}}\left[\tilde{f}_{\mathrm{pg}} \tilde{\boldsymbol{u}}+\tilde{\sigma}_{g}{ }^{2} \nabla_{\widetilde{\boldsymbol{u}}} \tilde{f}_{\mathrm{pg}}\right]=0 .
\end{aligned}
$$

In the rest of the paper, where only nondimensional variables are considered, the tildes will be systematically omitted for the sake of simplicity.

\section{Asymptotic models for large particles}

In this part, we consider the case of large particles observed at intermediate timescales. By intermediate time-scales, we mean that the ratio of the macroscopic observation time $T$ to the response time of the particles $\tau_{p}$ is supposed to be fixed, and we study the asymptotic behavior of the joint density $f_{\mathrm{pg}}$ when the Stokes number $S$ goes to infinity, or equivalently when $\tau_{p}$ is assumed to be much greater than the turbulence auto-correlation time $\tau_{g}$. In that case, we show by using a formal Chapman-Enskog expansion that the dependence of $f_{\mathrm{pg}}$ with respect on the gas turbulent velocity $\boldsymbol{u}$ can be explicitly computed (up to some terms of order $o(1 / S)$ ), which allows one to replace the original kinetic equation (2.3) by a drift-diffusion equation on the reduced phase space $\mathbb{R}_{\boldsymbol{x}}^{N} \times \mathbb{R}_{\boldsymbol{v}}^{N}$. More precisely, we have for the problem in nondimensional form:

Proposition 3.1 (Large particles at intermediate time-scale). Let $K$ fixed be given. For large values of $S$, the solution $f_{\mathrm{pg}}$ of $E q$. (2.12) satisfies:

$$
f_{\mathrm{pg}}(t, \boldsymbol{x}, \boldsymbol{v}, \boldsymbol{u})=\frac{1}{\left(2 \pi \bar{\sigma}_{g}^{2}\right)^{N / 2}} \exp \left(\frac{-\boldsymbol{u}^{2}}{2 \bar{\sigma}_{g}^{2}}\right)\left(f_{p}(t, \boldsymbol{x}, \boldsymbol{v})-\frac{1}{S} \boldsymbol{u} \cdot \nabla_{\boldsymbol{v}} f_{p}(t, \boldsymbol{x}, \boldsymbol{v})\right)+o\left(\frac{1}{S}\right),
$$

where $f_{p}$ depends only on $(t, \boldsymbol{x}, \boldsymbol{v})$ and satisfies the Fokker-Planck equation

$$
\partial_{t} \bar{f}_{p}+\operatorname{div}_{\boldsymbol{x}}\left(\boldsymbol{v} \bar{f}_{p}\right)+\frac{1}{K} \operatorname{div}_{\boldsymbol{v}}\left[-\boldsymbol{v} \bar{f}_{p}-\frac{\bar{\sigma}_{g}^{2}}{S} \nabla_{\boldsymbol{v}} \bar{f}_{p}\right]=0 .
$$

\section{REMARK 3.2.}

1 Coming back to the primitive dimensional variables, the result of Proposition 3.1 writes formally (for $S$ large enough):

$$
f_{\mathrm{pg}}=\frac{1}{\left(2 \pi \sigma_{g}^{2}\right)^{N / 2}} \exp \left(\frac{-\boldsymbol{u}^{2}}{2 \sigma_{g}^{2}}\right)\left(f_{p}-\frac{1}{S} \boldsymbol{u} \cdot \nabla_{\boldsymbol{v}} f_{p}\right)+o(1 / S),
$$

with $f_{p}$ satisfies the Fokker-Planck equation

$$
\partial_{t} f_{p}+\operatorname{div}_{\boldsymbol{x}}\left(\boldsymbol{v} f_{p}\right)+\operatorname{div}_{\boldsymbol{v}}\left[-\frac{\boldsymbol{v}}{\tau_{p}} f_{p}-\frac{\sigma_{g}^{2} \tau_{g}}{\tau_{p}^{2}} \nabla_{\boldsymbol{v}} f_{p}\right]=0 .
$$

2 This last equation is closely related to the one obtained by Reeks [25] or by Clouet and Domelevo in [4] and Goudon and Poupaud in [9] in the case of the so-called white-noise limit, which corresponds to the assumption (with the notation of the present paper): $\tau_{g} \rightarrow 0, \sigma_{g}^{2} \rightarrow+\infty$ with $\sigma_{g}^{2} \tau_{g}$ constant. Note that in [4], the authors found the diffusion coefficient (with respect to 
the variable $\boldsymbol{v}$ ) to be $D_{\boldsymbol{v}}=\frac{\sigma_{g}^{2} \tau_{g}}{2 \tau_{p}^{2}}$ instead of $D_{\boldsymbol{v}}=\frac{\sigma_{g}^{2} \tau_{g}}{\tau_{p}^{2}}$ as in the present work. This is in full agreement with the fact that, in their case, the stochastic process used to sample the turbulent gas velocity along the particle path is not the Langevin equation (2.2) but the so-called eddy-life-time model [7], whose integral auto-correlation time is not $\tau_{g}$ but $\frac{\tau_{g}}{2}$.

3 We note that the leading term in the formal expansion of $f_{\mathrm{pg}}$ writes:

$$
f_{\mathrm{pg}}^{0}(t, \boldsymbol{x}, \boldsymbol{v}, \boldsymbol{u})=\frac{1}{\left(2 \pi \sigma_{g}^{2}\right)^{N / 2}} \exp \left(\frac{-\boldsymbol{u}^{2}}{2 \sigma_{g}^{2}}\right) f_{p}(t, \boldsymbol{x}, \boldsymbol{v}) .
$$

This relation shows that, up to a term of order $1 / S$, the particle and the gas velocity at the same point are uncorrelated, which is quite evident from a physical point of view since, in this limit, the turbulent time-scale is supposed to be very small compared to the particle response time. Note that, if we take into account the first order term in the expansion of $f_{\mathrm{pg}}$, we obtain the following expression for the correlation tensor between gas and particle velocities:

$$
\frac{1}{N_{p}} \int_{\mathbb{E}} \boldsymbol{u} \otimes \boldsymbol{v} f_{\mathrm{pg}}(t, \boldsymbol{x}, \boldsymbol{v}, \boldsymbol{u}) \mathrm{d} \boldsymbol{x} \mathrm{d} \boldsymbol{v} \mathrm{d} \boldsymbol{u}=\frac{\sigma_{g}^{2}}{S} \boldsymbol{I}+o(1 / S),
$$

where $N_{p}$ is the total number of particles, which is in agreement with Tchen's formula (see [30])

$$
\frac{1}{N_{p}} \int_{\mathbb{E}} \boldsymbol{u} \otimes \boldsymbol{v} f_{\mathrm{pg}}(t, \boldsymbol{x}, \boldsymbol{v}, \boldsymbol{u}) \mathrm{d} \boldsymbol{x} \mathrm{d} \boldsymbol{v} \mathrm{d} \boldsymbol{u}=\frac{\sigma_{g}^{2}}{1+S} \boldsymbol{I}
$$

in the limit $S \rightarrow+\infty$.

Proof of Proposition 3.1. Introductory remarks. Recall briefly the general procedure for the derivation of the reduced model of Proposition 3.1 (see e.g. [2, 3, $1,6]$ ). In the case $S>1$, the nondimensional equation (2.12) writes as

$$
\partial_{t} f_{\mathrm{pg}}^{S}+\operatorname{div}_{\boldsymbol{x}}\left(\boldsymbol{v} f_{\mathrm{pg}}^{S}\right)+\frac{1}{K} \operatorname{div}_{\boldsymbol{v}}\left[(\boldsymbol{u}-\boldsymbol{v}) f_{\mathrm{pg}}^{S}\right]-\frac{S}{K} \mathcal{A}_{\boldsymbol{u}}\left(f_{\mathrm{pg}}^{S}\right)=0
$$

where the operator $\mathcal{A}_{\boldsymbol{u}}$ is defined as

$$
\mathcal{A}_{\boldsymbol{u}}(f):=\operatorname{div}_{\boldsymbol{u}}\left(\boldsymbol{u} f+\sigma_{g}^{2} \nabla_{\boldsymbol{u}} f\right) .
$$

In particular, when $S \gg 1$, the equation for $f_{\mathrm{pg}}$ above reduces formally to $\mathcal{A}_{\boldsymbol{u}}\left(f_{\mathrm{pg}}^{S}\right)=$ $O(1 / S)$. Hence, when $S \rightarrow+\infty$, we formally get that $f_{\mathrm{pg}}^{S} \rightarrow f_{\mathrm{pg}}^{0}$ where $f_{\mathrm{pg}}^{0}$ is solution of $\mathcal{A}_{\boldsymbol{u}}\left(f_{\mathrm{pg}}^{0}\right)=0$. It can be easily checked that the unique bounded positive solution to the latter equation that has the same zeroth order moment w.r.t. $\boldsymbol{u}$ as $f_{\mathrm{pg}}^{0}$ writes as

$$
f_{\mathrm{pg}}^{0}(t, \boldsymbol{x}, \boldsymbol{v}, \boldsymbol{u})=\frac{1}{\left(2 \pi \bar{\sigma}_{g}^{2}\right)^{N / 2}} \exp \left(\frac{-\boldsymbol{u}^{2}}{2 \bar{\sigma}_{g}^{2}}\right) f_{p}^{0}(t, \boldsymbol{x}, \boldsymbol{v}),
$$

where $f_{p}^{0}(t, \boldsymbol{x}, \boldsymbol{v})$ is defined as

$$
f_{p}^{0}(t, \boldsymbol{x}, \boldsymbol{v}) \stackrel{\text { def }}{=} \int_{\boldsymbol{u}} f_{\mathrm{pg}}^{0}(t, \boldsymbol{x}, \boldsymbol{v}, \boldsymbol{u}) \mathrm{d} \boldsymbol{u} .
$$


This suggests to look for an evolution equation for the zeroth order moment of $f_{\mathrm{pg}}^{S}$ with respect to $\boldsymbol{u}$, that is for $f_{p}^{S}(t, \boldsymbol{x}, \boldsymbol{v}):=\int_{\boldsymbol{u}} f_{\mathrm{pg}}^{S}(t, \boldsymbol{x}, \boldsymbol{v}, \boldsymbol{u}) \mathrm{d} \boldsymbol{u}$. Notice here that this moment is still a probability density but defined on the reduced phase space $\mathbb{R}_{\boldsymbol{x}}^{N} \times \mathbb{R}_{\boldsymbol{v}}^{N}$. Namely, integrating the equation (3.2) for $f_{\mathrm{pg}}^{S}$ against the variable $\boldsymbol{u}$ gives, for any $S>1$,

$$
\partial_{t} f_{p}^{S}+\operatorname{div}_{\boldsymbol{x}}\left(\boldsymbol{v} f_{p}^{S}\right)+\frac{1}{K} \operatorname{div} \boldsymbol{v}\left[-\boldsymbol{v} f_{p}^{S}+\int_{\boldsymbol{u}} \boldsymbol{u} f_{\mathrm{pg}}^{S} \mathrm{~d} \boldsymbol{u}\right]=0
$$

where the contribution of the operator $\mathcal{A}_{\boldsymbol{u}}$ disappears, and where we defined

$$
f_{p}^{S}(t, \boldsymbol{x}, \boldsymbol{v}) \stackrel{\text { def }}{=} \int_{\boldsymbol{u}} f_{\mathrm{pg}}^{S}(t, \boldsymbol{x}, \boldsymbol{v}, \boldsymbol{u}) \mathrm{d} \boldsymbol{u} .
$$

Equation (3.4) is in closed form in the limit $1 / S=0$. Indeed, since formally $f_{\mathrm{pg}}^{S}=$ $f_{\mathrm{pg}}^{0}+O(1 / S)$, we have for all $S>0$ that

$$
\partial_{t} f_{p}^{S}+\operatorname{div}_{\boldsymbol{x}}\left(\boldsymbol{v} f_{p}^{S}\right)+\frac{1}{K} \operatorname{div}_{\boldsymbol{v}}\left(-\boldsymbol{v} f_{p}^{S}\right)=O(1 / S)
$$

because the first order moment w.r.t. $\boldsymbol{u}$ of $f_{\mathrm{pg}}^{0}$ vanishes. Omitting the error term in the right-hand-side, we are facing a transport equation where no diffusion phenomenon is present. At the light of the preceding remarks, the Chapman-Enskog procedure allows one to estimate the error term in the equation above and to obtain a convectiondiffusion equation for the problem at hand.

\section{Chapman-Enskog expansion.}

Here, we want to estimate $f_{\mathrm{pg}}$ in terms of a first order expansion. For that, for all $S>0$, let us introduce the functions $f_{p}^{S}$ and $g_{\mathrm{pg}}^{S}$ such that, by definition:

$$
f_{\mathrm{pg}}^{S}=\frac{1}{\left(2 \pi \sigma_{g}^{2}\right)^{N / 2}} \exp \left(\frac{-\boldsymbol{u}^{2}}{2 \sigma_{g}^{2}}\right) f_{p}^{S}+\frac{1}{S} g_{\mathrm{pg}}^{S},
$$

with

$$
f_{p}^{S}(t, \boldsymbol{x}, \boldsymbol{v})=\int_{\mathbb{R}^{N}} f_{\mathrm{pg}}^{S} \mathrm{~d} \boldsymbol{u}, \quad \int_{\mathbb{R}^{N}} g_{\mathrm{pg}}^{S} \mathrm{~d} \boldsymbol{u}=0 .
$$

Injecting the ansatz (3.5) in Eq. (2.12) yields:

$$
\begin{aligned}
& \mathcal{A}_{\boldsymbol{u}}\left(g_{\mathrm{pg}}^{S}\right)= \frac{K}{\left(2 \pi \bar{\sigma}_{g}^{2}\right)^{N / 2}} \exp \left(\frac{-\boldsymbol{u}^{2}}{2 \bar{\sigma}_{g}^{2}}\right)\left[\partial_{t} f_{p}^{S}+\operatorname{div}_{\boldsymbol{x}}\left(\boldsymbol{v} f_{p}^{S}\right)+\operatorname{div} \boldsymbol{v}\left(\frac{\boldsymbol{u}-\boldsymbol{v}}{K} f_{p}^{S}\right)\right] \\
&+\frac{1}{S}\left[\partial_{t} g_{\mathrm{pg}}^{S}+\operatorname{div}_{\boldsymbol{x}}\left(\boldsymbol{v} g_{\mathrm{pg}}^{S}\right)+\operatorname{div}_{\boldsymbol{v}}\left(\frac{\boldsymbol{u}-\boldsymbol{v}}{K} g_{\mathrm{pg}}^{S}\right)\right] .
\end{aligned}
$$

By construction, we know that $f_{p}^{S} \rightarrow f_{p}^{0}$ when $S \rightarrow+\infty$. Therefore, thanks to the fact that $f_{p}^{0}$ satisfies $(3.3)$, it follows that $g_{\mathrm{pg}}^{S}$ tends formally to $g_{\mathrm{pg}}^{0}$ when $S \rightarrow+\infty$, where $g_{\mathrm{pg}}^{0}$ is a solution to:

$$
\mathcal{A}_{\boldsymbol{u}}\left(g_{\mathrm{pg}}^{0}\right)=\frac{1}{\left(2 \pi \bar{\sigma}_{g}^{2}\right)^{N / 2}} \exp \left(\frac{-\boldsymbol{u}^{2}}{2 \bar{\sigma}_{g}^{2}}\right) \boldsymbol{u} \cdot \nabla_{v} f_{p}^{0}, \int_{\mathbb{R}^{N}} g_{\mathrm{pg}}^{0} \mathrm{~d} \boldsymbol{u}=0
$$


The unique solution of this problem writes:

$$
g_{\mathrm{pg}}^{0}=\frac{-1}{\left(2 \pi \bar{\sigma}_{g}^{2}\right)^{N / 2}} \exp \left(\frac{-\boldsymbol{u}^{2}}{2 \bar{\sigma}_{g}^{2}}\right) \boldsymbol{u} \cdot \nabla_{v} f_{p}^{0}
$$

Since $f_{p}^{0}$ (respectively $g_{\mathrm{pg}}^{0}$ ) is the limit of $f_{p}^{S}$ (respectively $g_{\mathrm{pg}}^{S}$ ), when $S \rightarrow+\infty$, it follows that there exists (at least formally) a function $\varepsilon$ defined on $\left[0,+\infty\left[^{2} \times \mathcal{E}\right.\right.$; such that $\forall t>0, \forall \xi \in \mathcal{E}, \lim _{z \rightarrow 0} \varepsilon(z, t, \xi)=0$ and such that for all $S$ large enough:

$$
g_{\mathrm{pg}}^{S}=\frac{-1}{\left(2 \pi \bar{\sigma}_{g}^{2}\right)^{N / 2}} \exp \left(\frac{-\boldsymbol{u}^{2}}{2 \bar{\sigma}_{g}^{2}}\right) \boldsymbol{u} \cdot \nabla_{v} f_{p}^{S}+\varepsilon\left(\frac{1}{S}\right) .
$$

Inserting this expression into (3.5), we get:

$$
f_{\mathrm{pg}}=\frac{1}{\left(2 \pi \bar{\sigma}_{g}^{2}\right)^{N / 2}} \exp \left(\frac{-\boldsymbol{u}^{2}}{2 \bar{\sigma}_{g}^{2}}\right)\left(f_{p}^{S}-\frac{1}{S} \boldsymbol{u} \cdot \nabla_{v} f_{p}^{S}\right)+o\left(\frac{1}{S}\right) .
$$

Also, a short calculation yields:

$$
\int_{\mathbb{R}^{N}} \boldsymbol{u} f_{\mathrm{pg}} \mathrm{d} \boldsymbol{u}=\frac{-\bar{\sigma}_{g}^{2}}{S} \nabla_{v} f_{p}^{S}+o\left(\frac{1}{S}\right) .
$$

Hence, using $f_{p}^{S}=\int_{\mathbb{R}^{N}} f_{\mathrm{pg}} d \boldsymbol{u}$, we find that $f_{p}^{S}$ satisfies:

$$
\partial_{t} f_{p}^{S}+\operatorname{div}_{\boldsymbol{x}}\left(\boldsymbol{v} f_{p}^{S}\right)+\frac{1}{K} \operatorname{div}_{\boldsymbol{v}}\left[-\boldsymbol{v} f_{p}^{S}-\frac{\bar{\sigma}_{g}^{2}}{S} \nabla_{\boldsymbol{v}} f_{p}^{S}\right]=o\left(\frac{1}{S}\right) .
$$

Finally, let $f_{p}$ be the solution of $(3.1)$ :

$$
\partial_{t} f_{p}+\operatorname{div}_{\boldsymbol{x}}\left(\boldsymbol{v} f_{p}\right)+\frac{1}{K} \operatorname{div}_{\boldsymbol{v}}\left[-\boldsymbol{v} f_{p}-\frac{\bar{\sigma}_{g}^{2}}{S} \nabla_{\boldsymbol{v}} f_{p}\right]=0 .
$$

Formally, it follows that $f_{p}^{S}=f_{p}+o\left(\frac{1}{S}\right)$ and $\frac{1}{S} \nabla f_{p}^{S}=\frac{1}{S} \nabla f_{p}+o\left(\frac{1}{S}\right)$. Hence, inserting these relations in (3.6), we obtain:

$$
f_{\mathrm{pg}}=\frac{1}{\left(2 \pi \bar{\sigma}_{g}^{2}\right)^{N / 2}} \exp \left(\frac{-\boldsymbol{u}^{2}}{2 \bar{\sigma}_{g}^{2}}\right)\left(f_{p}-\frac{1}{S} \boldsymbol{u} \cdot \nabla_{v} f_{p}\right)+o\left(\frac{1}{S}\right)
$$

which is the desired result.

We now consider the asymptotic behaviour of the solutions of equation (2.12) when the observation time $T$ is much greater than the particle response time $\tau_{p}$. We have the following result:

Proposition 3.3 (Large particles at large time-scale). Let $f_{p}$ be given as in Proposition 3.1 above. For small values of $K$, the solution $f_{p}$ of $E q$. (3.1) satisfies:

$$
\bar{f}_{p}(t, \boldsymbol{x}, \boldsymbol{v})=\frac{S^{N / 2}}{\left(2 \pi \bar{\sigma}_{g}^{2}\right)^{N / 2}} \exp \left(\frac{-S \boldsymbol{v}^{2}}{2 \bar{\sigma}_{g}^{2}}\right)\left[\bar{n}_{p}(t, \boldsymbol{x})-K \boldsymbol{v} \cdot \nabla_{\boldsymbol{x}} \bar{n}_{p}(t, \boldsymbol{x})\right]+o(K),
$$


where $n_{p}$ depends only on $(t, \boldsymbol{x})$ and satisfies the diffusion equation

$$
\partial_{t} \bar{n}_{p}-\operatorname{div}_{\boldsymbol{x}}\left(\frac{K \bar{\sigma}_{g}^{2}}{S} \nabla_{\boldsymbol{x}} \bar{n}_{p}\right)=0 .
$$

REMARK 3.4. Coming back to the primitive dimensional variables, the result of proposition 3.3 writes formally (for $K$ small enough):

$$
f_{p}(t, \boldsymbol{x}, \boldsymbol{v})=\frac{S^{N / 2}}{\left(2 \pi \sigma_{g}^{2}\right)^{N / 2}} \exp \left(\frac{-S \boldsymbol{v}^{2}}{2 \sigma_{g}^{2}}\right)\left(n_{p}(t, \boldsymbol{x})-\tau_{p} \boldsymbol{v} \cdot \nabla_{\boldsymbol{x}} n_{p}(t, \boldsymbol{x})\right)+o(K),
$$

with $n_{p}$ satisfies the diffusion equation

$$
\partial_{t} n_{p}-\operatorname{div}_{\boldsymbol{x}}\left(\sigma_{g}^{2} \tau_{g} \nabla_{\boldsymbol{x}} n_{p}\right)=0 .
$$

Proof of Proposition 3.3. The proof follows exactly the same lines as the proof of proposition 3.1. For the sake of completeness, we provide the details in Appendix B.

\section{Asymptotic models for small particles}

We consider now the case where the particles are very small, that is when $\tau_{p} \ll \tau_{g}$, or equivalently $S \ll 1$. Small particles therefore tend to behave like fluid particles, i.e. passive tracers. We are interested here in reduced models of the joint pdf $f_{\mathrm{pg}}$ in two situtations. First, as done before, we derive an asymptotic model in the case where we observe the system at time-scales of order $\tau_{g}$. In that first limit, the turbulent gas flow is not at equilibrium, in contrast with the particles, which reach a partial equilibrium thanks to the drag force. We obtain here an asymptotic model for the distribution of the velocity of the gas along the paths of the particles. As a second step, we consider the limit where the observation time is much larger than the turbulent correlation time, and we obtain the same diffusion equation as in the previous section. This shows again that the macroscopic rate of diffusion of particles in a prescribed turbulence seen by the particles is independent of the size of the particles (see $[30,10]$ ).

Proposition 4.1 (Small particles at intermediate time-scale). Let $K$ fixed be given. For $S$ small enough, we have:

$$
f_{\mathrm{pg}}(t, \boldsymbol{x}, \boldsymbol{v}, \boldsymbol{u})=\frac{1}{\left(2 \pi S \bar{\sigma}_{g}^{2}\right)^{N / 2}} \exp \left(\frac{-(\boldsymbol{v}-\boldsymbol{u})^{2}}{2 S \bar{\sigma}_{g}^{2}}\right) f_{g}(t, \boldsymbol{x}, \boldsymbol{u})+O(\sqrt{S}),
$$

where $f_{g}$ depends only on $(t, \boldsymbol{x}, \boldsymbol{u})$ and obeys the Fokker-Planck equation

$$
\partial_{t} f_{g}+\operatorname{div}_{\boldsymbol{x}}\left(\boldsymbol{u} f_{g}\right)+\frac{1}{K} \operatorname{div}_{\boldsymbol{u}}\left(-\boldsymbol{u} f_{g}-\sigma_{g}^{2} \nabla_{\boldsymbol{u}} f_{g}\right)=0 .
$$

The reader is invited to check that the corresponding dimensional form is indeed that stated in section 2, namely Eq. (2.6)-(2.7).

Proof. We start again with the nondimensional equation for the joint pdf $f_{\mathrm{pg}}^{S}$ in the case $S \leq 1$ :

$$
\partial_{t} \bar{f}_{\mathrm{pg}}^{S}+\operatorname{div}_{\boldsymbol{x}}\left(\boldsymbol{v} \bar{f}_{\mathrm{pg}}^{S}\right)+\frac{1}{S K} \operatorname{div}_{\boldsymbol{v}}\left[(\boldsymbol{u}-\boldsymbol{v}) \bar{f}_{\mathrm{pg}}^{S}\right]-\frac{1}{K} \operatorname{div}_{\boldsymbol{u}}\left[\boldsymbol{u} \bar{f}_{\mathrm{pg}}^{S}+\bar{\sigma}_{g}^{2} \nabla_{\boldsymbol{u}} \bar{f}_{\mathrm{pg}}^{S}\right]=0 .
$$


One should notice here that the limiting model obtained for $S \ll 1$ is singular in the sense that the distribution of particles' velocities tends to a Dirac measure centered around the gas velocity $\boldsymbol{u}$. In order to tackle this difficulty, the proof of Proposition 4.1 consists of the following steps:

1. find a new formulation of the problem where no singular limiting equilibrium distributions are present,

2. seek for a reduced model in the frame of this new formulation.

3. recast the problem in terms of the original unknowns and variables.

We will see that Step 2 is simpler than in the previous cases.

Step 1: getting rid of the singularity. For any small but positive values of $S$, we can perform the change of variables:

$$
\begin{aligned}
f_{\mathrm{pg}}^{S}(t, \boldsymbol{x}, \boldsymbol{v}, \boldsymbol{u}) & :=\frac{1}{\sqrt{S}^{N}} f_{1}^{S}\left(t, \boldsymbol{x}, \frac{\boldsymbol{v}-\boldsymbol{u}}{\sqrt{S}}, \boldsymbol{u}\right):=\frac{1}{\sqrt{S}^{N}} f_{1}^{S}\left(t, \boldsymbol{x}, \boldsymbol{v}_{1}, \boldsymbol{u}_{1}\right) \\
\text { where } \quad \boldsymbol{v}_{1} & =\frac{\boldsymbol{v}-\boldsymbol{u}}{\sqrt{S}}, \quad \text { and } \quad \boldsymbol{u}_{1}=\boldsymbol{u},
\end{aligned}
$$

which amounts to zooming on the singularity at a scale where the drag force and the turbulent dispersion effects are of the same order. Indeed, straightforward calculations show that the new unknown $f_{1}^{S}$ solves:

$$
\begin{aligned}
\partial_{t} f_{1}^{S} & +\operatorname{div}_{\boldsymbol{x}}\left(\boldsymbol{u}_{1} f_{1}^{S}\right)+\frac{1}{K} \operatorname{div}_{\boldsymbol{u}_{1}}\left(-\boldsymbol{u}_{1} f_{1}^{S}-\bar{\sigma}_{g}^{2} \nabla_{\boldsymbol{u}_{1}} f_{1}^{S}\right)+\sqrt{S} \operatorname{div}_{\boldsymbol{x}}\left(\boldsymbol{v}_{1} f_{1}^{S}\right) \\
& +\frac{1}{K \sqrt{S}} \operatorname{div}_{\boldsymbol{v}_{1}}\left(\boldsymbol{u}_{1} f_{1}^{S}+2 \bar{\sigma}_{g}^{2} \nabla_{\boldsymbol{u}_{1}} f_{1}^{S}\right)+\frac{1}{S K} \operatorname{div}_{\boldsymbol{v}_{1}}\left(-\boldsymbol{v}_{1} f_{1}^{S}-\bar{\sigma}_{g}^{2} \nabla_{\boldsymbol{v}_{1}} f_{1}^{S}\right)=0
\end{aligned}
$$

where the last term is of leading order. The scaling used here in (4.1) is similar to the scaling used in the papers of Jabin $[12,13]$ as well as the paper of Goudon, Jabin and Vasseur [8].

Step 2: reduced model. The important feature of the analysis is that a relevant reduced model involving the diffusion phenomenon is easily obtained as a 0 -th order approximation of Eq. (4.2) above. Indeed, observe first that defining as usual

$$
\begin{aligned}
f_{1}^{S}\left(t, \boldsymbol{x}, \boldsymbol{v}_{1}, \boldsymbol{u}_{1}\right) & =\frac{1}{\left(2 \pi \bar{\sigma}_{g}^{2}\right)^{N / 2}} \exp \left(-\frac{\boldsymbol{v}_{1}^{2}}{2 \bar{\sigma}_{g}}\right) f_{1 g}^{S}\left(t, \boldsymbol{x}, \boldsymbol{u}_{1}\right)+\sqrt{S} g_{1}^{S}\left(t, \boldsymbol{x}, \boldsymbol{v}_{1}, \boldsymbol{u}_{1}\right), \\
\text { where } f_{1 g}^{S}\left(t, \boldsymbol{x}, \boldsymbol{u}_{1}\right): & =\int_{\boldsymbol{v}_{1}} f_{1}^{S}\left(t, \boldsymbol{x}, \boldsymbol{v}_{1}, \boldsymbol{u}_{1}\right) \mathrm{d} \boldsymbol{v}_{1}, \quad \text { and consequently } \int_{\boldsymbol{v}_{1}} g_{1}^{S} \mathrm{~d} \boldsymbol{v}_{1}=0 .
\end{aligned}
$$

A direct integration of Eq. (4.2) with respect to the variable $\boldsymbol{v}_{1}$ together with the definition of $g_{1}^{S}$ yields formally the diffusion equation for $f_{1 g}^{S}$ :

$$
\begin{aligned}
& \partial_{t} f_{1 g}^{S}+\operatorname{div}_{\boldsymbol{x}}\left(\boldsymbol{u}_{1} f_{1 g}^{S}\right)+\frac{1}{K} \operatorname{div} \boldsymbol{u}_{1}\left(-\boldsymbol{u}_{1} f_{1 g}^{S}-\sigma_{g}^{2} \nabla_{\boldsymbol{u}_{1}} f_{1 g}^{S}\right)=-\sqrt{S} \operatorname{div}_{\boldsymbol{x}}\left(\int_{\boldsymbol{v}_{1}} \boldsymbol{v}_{1} f_{1}^{S} \mathrm{~d} \boldsymbol{v}_{1}\right) \\
& =-\sqrt{S} \operatorname{div}_{\boldsymbol{x}}\left(\int_{\boldsymbol{v}_{1}} \boldsymbol{v}_{1} \sqrt{S} g_{1}^{S} \mathrm{~d} \boldsymbol{v}_{1}\right)=O(S) .
\end{aligned}
$$

Hence formally $f_{1 g}^{S}=f_{1 g}+O(S)$, where $f_{1 g}$ is solution to

$$
\partial_{t} f_{1 g}+\operatorname{div}_{\boldsymbol{x}}\left(\boldsymbol{u}_{1} f_{1 g}\right)+\frac{1}{K} \operatorname{div} \boldsymbol{u}\left(-\boldsymbol{u}_{1} f_{1 g}-\sigma_{g}^{2} \nabla_{\boldsymbol{u}} f_{1 g}\right)=0 .
$$


Step 3: coming back to the original unknowns and variables. Coming back to the original unknowns and variables, that is successively $f_{1}^{S} \rightarrow f_{\mathrm{pg}}^{S}, f_{1 g}^{S} \rightarrow f_{g}^{S}$, $f_{1 g} \rightarrow f_{g}$, and $\left(\boldsymbol{v}_{1}, \boldsymbol{u}_{1}\right) \rightarrow(\boldsymbol{v}, \boldsymbol{u})$, yields the result of the proposition.

REMARK 4.2. It is important to understand that though the variable $\boldsymbol{v}$ for the velocity of the particles does not appear any longer in the problem, the pdf $f_{g}$ still describes the motion of the inertial particles. Recall indeed that the variable $\boldsymbol{u}$ stands for the velocity of the gas seen by a particle along its trajectory. In the case of asymptotically small particles, the drag force is so strong that the particles are expected to behave as passive scalars moving at the velocity of the gas, which is indeed the case as seen from the transport term $\operatorname{div}_{\boldsymbol{x}}\left(\boldsymbol{u} f_{g}\right)$. In other words, the equation for the pdf $f_{g}(t, \boldsymbol{x}, \boldsymbol{u})$ is a shorthand for the system of ODEs $\mathrm{d} \boldsymbol{x}_{p} / \mathrm{d} t=\boldsymbol{u}_{p}$ and $\mathrm{d} \boldsymbol{u}_{p}=-\boldsymbol{u}_{p} / \tau_{g} \mathrm{~d} t+\sqrt{2 \sigma_{g}^{2} / \tau_{g}} \mathrm{~d} \mathbf{W}_{\mathbf{t}}$.

Then, notice that in our approach the probable turbulent velocity $\boldsymbol{u}_{p}(t)$ seen by the particles is not necessarily gaussian for all time. It is only after a time $T \gg \tau_{g}$ that one expects such a property (hence Proposition 4.3 below). Conversely, for an observation time $T \sim \tau_{g}$ as in Proposition 4.1 above, we have to consider the transient regime towards gaussianity with respect to the variable $\boldsymbol{u}$. In particular it is not possible to apply in this regime Reeks' formula (1.5) as an alternative means for computing the value of the diffusion coefficient of the reduced model.

Now, for large values of the time $T$ of observation, one has the result:

Proposition 4.3 (Small particles at large time-scale). Let $f_{g}$ be the solution of proposition 4.1 above. Then, when $K$ goes to zero, we have:

$$
f_{g}(t, \boldsymbol{x}, \boldsymbol{u})=\frac{1}{\left(2 \pi \bar{\sigma}_{g}^{2}\right)^{N / 2}} \exp \left(-\frac{\boldsymbol{u}^{2}}{\bar{\sigma}_{g}^{2}}\right)\left[n_{p}(t, \boldsymbol{x})-K \boldsymbol{u} . \nabla_{\boldsymbol{x}} n_{p}(t, \boldsymbol{x})\right]+\varepsilon(K),
$$

where $n_{p}$ depends only on $(t, \boldsymbol{x})$ and satisfies the diffusion equation

$$
\partial_{t} \bar{n}_{p}-\operatorname{div}_{\boldsymbol{x}}\left(K \bar{\sigma}_{g}^{2} \nabla_{\boldsymbol{x}} \bar{n}_{p}\right)=0 .
$$

Proof. The proof follows exactly the same lines as the corresponding results in the previous two sections. The corresponding dimensional form is Eq. (2.8).

\section{Asymptotic model for particles with finite inertia}

In this part, we now consider the case of particles with finite inertia, corresponding to the hypothesis that $S$ is a fixed constant. Assuming that the observation time-scale is much greater than both $\tau_{p}$ and $\tau_{g}$, we first show that the asymptotic limit of the joint particle-gas velocity pdf is a correlated normal law. Taking into account the first order corrective term, we also prove formally that the particle concentration $n_{p}=$ $\int_{\mathbb{R}^{2 N}} f_{\mathrm{pg}} d \boldsymbol{v} d \boldsymbol{u}$ satisfies the diffusion equation (3.7) already obtained above for both large and light particles. This paradoxical property can be explained qualitatively by the fact that large particles have a lower mean square velocity than light ones but a larger auto-correlation time-scale and therefore follow less erratic trajectories.

Proposition 5.1 (particles of finite inertia at large time-scale). Let $S$ fixed be given. When $K$ goes to zero, we have:

$$
\begin{aligned}
\bar{f}_{\mathrm{pg}}(t, \boldsymbol{x}, \boldsymbol{v}, \boldsymbol{u})= & \mathcal{M}_{\mathrm{pg}}\left(\boldsymbol{v}, \boldsymbol{u} ; \sigma_{g}, S\right)\left(\bar{n}_{p}(t, \boldsymbol{x})-\frac{K}{\max (1, S)}\left(\frac{\boldsymbol{u}}{S}+(2+1 / S) \boldsymbol{v}\right) \cdot \nabla_{\boldsymbol{x}} \bar{n}_{p}(t, \boldsymbol{x})\right) \\
& +o(K)
\end{aligned}
$$


where $\mathcal{M}_{\mathrm{pg}}$ is defined as the correlated normal law:

$$
\mathcal{M}_{\mathrm{pg}}(\boldsymbol{v}, \boldsymbol{u})=\left[\frac{(1+S)}{2 \pi \bar{\sigma}_{g}^{2} \sqrt{S}}\right]^{N} \exp \left[-\frac{1+S}{2 \bar{\sigma}_{g}^{2} S}\left(\boldsymbol{u}^{2}+(1+S) \boldsymbol{v}^{2}-2 \boldsymbol{u} \cdot \boldsymbol{v}\right)\right],
$$

and where $n_{p}$ depends only on $(t, \boldsymbol{x})$ and satisfies the diffusion equation

$$
\partial_{t} \bar{n}_{p}-\operatorname{div}_{\boldsymbol{x}}\left(\frac{K \bar{\sigma}_{g}^{2}}{\max (1, S)} \nabla_{\boldsymbol{x}} \bar{n}_{p}\right)=0
$$

Notice that the covariance matrix of $\mathcal{M}_{\mathrm{pg}}(\boldsymbol{v}, \boldsymbol{u})$ is

$$
\boldsymbol{\Sigma}:=\boldsymbol{\Sigma}\left(\sigma_{g}, S\right):=\left[\begin{array}{cc}
\mathbb{E}^{\omega}\left[\boldsymbol{v}_{p}^{2}\right] & \mathbb{E}^{\omega}\left[\boldsymbol{v}_{p} \boldsymbol{u}_{p}\right] \\
\mathbb{E}^{\omega}\left[\boldsymbol{v}_{p} \boldsymbol{u}_{p}\right] & \mathbb{E}^{\omega}\left[\boldsymbol{u}_{p}^{2}\right]
\end{array}\right]=\left[\begin{array}{cc}
\frac{\sigma_{g}^{2}}{1+S} & \frac{\sigma_{g}^{2}}{1+S} \\
\frac{\sigma_{g}^{2}}{1+S} & \sigma_{g}^{2}
\end{array}\right]
$$

REMARK 5.2. Coming back to the primitive dimensional variables, the result of proposition 5.1 writes formally:

$$
f_{\mathrm{pg}}(t, \boldsymbol{x}, \boldsymbol{v}, \boldsymbol{u})=\mathcal{M}(\boldsymbol{v}, \boldsymbol{u})\left(n_{p}(t, \boldsymbol{x})-\tau_{g}\left(\frac{\boldsymbol{u}}{S}+\left(\frac{2}{S}+1\right) \boldsymbol{v}\right) \cdot \nabla_{\boldsymbol{x}} n_{p}(t, \boldsymbol{x})\right)+o(K),
$$

where $n_{p}$ satisfies the drift-diffusion equation (see Eq. (2.8)):

$$
\partial_{t} n_{p}-\operatorname{div}_{\boldsymbol{x}}\left(\sigma_{g}^{2} \tau_{g} \nabla_{x} n_{p}\right)=0 .
$$

We note that this equation is exactly the same as (3.9) for large particles or (3.7) for small particles, both observed at large time-scale. In particular, the diffusion coefficient $D_{x}=\sigma_{g}^{2} \tau_{g}$ does not depend on the particle response time $\tau_{p}$ but is equal to the dispersion coefficient of the gas particles. As already mentioned above, this result was first obtained by Tchen [30] and Hinze [10] by applying spectral analysis techniques to the particle motion equations and using the correspondance between the dispersion coefficient and the particle velocity autocorrelation function $R(t)=$ $\mathbb{E}^{\omega}\left[\boldsymbol{v}_{p}(0) \boldsymbol{v}_{p}(t)\right]$

Proof of Proposition 5.1. Since the method of proof is the same as for proposition 3.1 , we only indicate the main steps and skip all the details. First, we note that $(2.12)$ yields:

$$
\mathcal{A}_{\boldsymbol{v} \boldsymbol{u}}\left(f_{\mathrm{pg}}\right)=\frac{K}{\max (1, S)}\left[\partial_{t} f_{\mathrm{pg}}+\operatorname{div}_{\boldsymbol{x}}\left(f_{\mathrm{pg}} \boldsymbol{v}\right)\right],
$$

where the operator $\mathcal{A}_{\boldsymbol{v} \boldsymbol{u}}$ is defined as:

$$
\mathcal{A}_{\boldsymbol{v} \boldsymbol{u}}(f)=\frac{1}{S} \operatorname{div}_{\boldsymbol{v}}[(\boldsymbol{u}-\boldsymbol{v}) f]-\operatorname{div} \boldsymbol{u}\left[\boldsymbol{u} f+\sigma_{g}^{2} \boldsymbol{\nabla}_{\boldsymbol{u}} f\right] .
$$

Hence, when $K \rightarrow 0^{+}$, we formally get that $f_{\mathrm{pg}} \rightarrow f_{\mathrm{pg}}^{0}$ where $f_{\mathrm{pg}}^{0}$ satisfies:

$$
\left\{\begin{array}{l}
(i) \quad \mathcal{A}_{\boldsymbol{v} \boldsymbol{u}}\left(f_{\mathrm{pg}}^{0}\right)=0 \\
(i i) \int_{\mathbb{R}^{2 N}} f_{\mathrm{pg}}^{0} \mathrm{~d} \boldsymbol{v} \mathrm{d} \boldsymbol{u}=n_{p}^{0} \\
(\text { iii }) \partial_{t} n_{p}^{0}+\operatorname{div}_{\boldsymbol{x}}\left(\int_{\mathbb{R}^{2 N}} \boldsymbol{v} f_{\mathrm{pg}}^{0} \mathrm{~d} \boldsymbol{v} \mathrm{d} \boldsymbol{u}\right)=0 .
\end{array}\right.
$$


It can be checked that:

LEMmA 5.3. The unique bounded positive solution of (i), under the constraint (ii), writes:

$$
f_{\mathrm{pg}}^{0}=n_{p}^{0} \mathcal{M}_{\mathrm{pg}}
$$

where $\mathcal{M}_{\text {pg }}$ is a correlated normal law defined as:

$$
\mathcal{M}_{\mathrm{pg}}(u, v)=\frac{1}{\left(2 \pi \bar{\sigma}_{g} \bar{\sigma}_{p}\right)^{N}\left(1-\xi_{\boldsymbol{u} \boldsymbol{v}}^{2}\right)^{N / 2}} \exp \left[\frac{-1}{\left(1-\xi_{\boldsymbol{u} \boldsymbol{v}}^{2}\right)}\left(\frac{\boldsymbol{u}^{2}}{2 \bar{\sigma}_{g}^{2}}+\frac{\boldsymbol{v}^{2}}{2 \bar{\sigma}_{p}^{2}}-2 \xi_{\boldsymbol{u v}} \frac{\boldsymbol{u} \cdot \boldsymbol{v}}{\bar{\sigma}_{g} \bar{\sigma}_{p}}\right)\right]
$$

with $\xi_{u \boldsymbol{v}}=\frac{1}{\sqrt{1+S}}$ and $\sigma_{p}=\frac{\bar{\sigma}_{g}}{\sqrt{1+S}}$.

Proof. The important part of the Lemma consists in proving uniqueness, since the second order operator $\mathcal{A}_{\boldsymbol{v} \boldsymbol{u}}$ is not strongly elliptic. (There are no second order derivatives with respect to the variable $\boldsymbol{v}$.) Here, the uniqueness property is obtained through the expression of the dissipation rate (see the appendix):

$$
\begin{aligned}
& \int_{\mathbb{R}^{2 N}} \mathcal{A}_{\boldsymbol{v} \boldsymbol{u}}\left(f_{\mathrm{pg}}\right) \ln \left(f_{\mathrm{pg}}\right) \mathrm{d} \boldsymbol{v} \mathrm{d} \boldsymbol{u} \\
= & \int_{\mathbb{R}^{2 N}} 4 \mathcal{M}_{\mathrm{pg}}\left(\nabla_{u} \sqrt{\frac{f_{\mathrm{pg}}}{\mathcal{M}_{\mathrm{pg}}}} \cdot \nabla_{u} \sqrt{\frac{f_{\mathrm{pg}}}{\mathcal{M}_{\mathrm{pg}}}}\right) \mathrm{d} \boldsymbol{v} \mathrm{d} \boldsymbol{u} \\
& +\frac{1+S}{S}\left[N \int_{\mathbb{R}^{2 N}} f_{\mathrm{pg}} \mathrm{d} \boldsymbol{v} \mathrm{d} \boldsymbol{u}-\int_{\mathbb{R}^{2 N}} \frac{1+S}{S} \frac{|\boldsymbol{v}-\boldsymbol{u}|^{2}}{\bar{\sigma}_{g}^{2}} f_{\mathrm{pg}} \mathrm{d} \boldsymbol{v} \mathrm{d} \boldsymbol{u}\right] .
\end{aligned}
$$

This kind of entropy estimate is rather classical in the mathematical theory of kinetic equations. Let $f$ be any positive solution of the problem $(i)-(i i)$. Let us prove that $f=n^{0} \mathcal{M}_{\mathrm{pg}}$. First, it follows from equation $(i)$ and equality (5.4) that $f$ must satisfy:

$$
\begin{aligned}
\int_{\mathbb{R}^{2 N}} 4 \mathcal{M}_{\mathrm{pg}}\left(\nabla_{u} \sqrt{\frac{f}{\mathcal{M}_{\mathrm{pg}}}} \cdot \nabla_{u} \sqrt{\frac{f}{\mathcal{M}_{\mathrm{pg}}}}\right) \mathrm{d} \boldsymbol{v} \mathrm{d} \boldsymbol{u} \\
+\frac{1+S}{S}\left[N \int_{\mathbb{R}^{2 N}} f \mathrm{~d} \boldsymbol{v} \mathrm{d} \boldsymbol{u}-\int_{\mathbb{R}^{2 N}} \frac{1+S}{S} \frac{|\boldsymbol{v}-\boldsymbol{u}|^{2}}{\bar{\sigma}_{g}^{2}} f \mathrm{~d} \boldsymbol{v} \mathrm{d} \boldsymbol{u}\right]=0 .
\end{aligned}
$$

Moreover, integrating $(i)$ with respect to $\boldsymbol{u}$ and $\boldsymbol{v}$ and using (ii), we easily obtain that

$$
\begin{gathered}
\int_{\mathbb{R}^{2 N}}|\boldsymbol{u}|^{2} f \mathrm{~d} \boldsymbol{v} \mathrm{d} \boldsymbol{u}=N n^{0} \bar{\sigma}_{g}^{2} \\
\int_{\mathbb{R}^{2 N}}|\boldsymbol{v}|^{2} f \mathrm{~d} \boldsymbol{v} \mathrm{d} \boldsymbol{u}=\int_{\mathbb{R}^{2 N}} \boldsymbol{v} \cdot \boldsymbol{u} f \mathrm{~d} \boldsymbol{v} \mathrm{d} \boldsymbol{u}=\frac{N}{1+S} n^{0} \bar{\sigma}_{g}^{2},
\end{gathered}
$$

so that

$$
N \int_{\mathbb{R}^{2 N}} f \mathrm{~d} \boldsymbol{v} \mathrm{d} \boldsymbol{u}-\int_{\mathbb{R}^{2 N}} \frac{1+S}{S} \frac{|\boldsymbol{v}-\boldsymbol{u}|^{2}}{\bar{\sigma}_{g}^{2}} f \mathrm{~d} \boldsymbol{v} \mathrm{d} \boldsymbol{u}=0
$$


Combining this last equality with (5.5), it follows that there exists a function $h:=h(\boldsymbol{v})$, which does not depend on $\boldsymbol{u}$, such that $f=h \mathcal{M}_{\mathrm{pg}}$. Using again that $f$ solves equation (i), a straightforward calculation yields:

$$
\forall(\boldsymbol{u}, \boldsymbol{v}) \in \mathbb{R}^{2 N},(\boldsymbol{v}-\boldsymbol{u}) \cdot \nabla_{\boldsymbol{v}} h=0
$$

which, combined with $(i i)$, gives $h=n^{0}$. This concludes the proof of the Lemma.

Let us come back now to the proof of Proposition 5.1. The relation (iii) and (5.3) show that $n_{p}^{0}$ must solve:

$$
\partial_{t} n_{p}^{0}=0
$$

Now, let $n_{p}^{K}$ and $g_{p}^{K}$ be such that:

$$
n_{p}^{K}=\int_{\mathbb{R}^{2 N}} f_{\mathrm{pg}} \mathrm{d} \boldsymbol{v} \mathrm{d} \boldsymbol{u}, \quad \int_{\mathbb{R}^{2 N}} g_{p}^{K} \mathrm{~d} \boldsymbol{u} \mathrm{d} \boldsymbol{v}=0,
$$

and

$$
f_{\mathrm{pg}}=\mathcal{M}_{\mathrm{pg}} n_{p}^{K}+K g_{p}^{K}
$$

Inserting this expression in equation (2.12) and using that $n_{p}^{K} \rightarrow n_{p}^{0}$, a solution of (5.6), when $K \rightarrow 0^{+}$, it follows that $g_{p}^{K} \rightarrow g_{p}^{0}$ with $g_{p}^{0}$ a solution of:

$$
\mathcal{A}_{\boldsymbol{u} \boldsymbol{v}}\left(g_{p}^{0}\right)=-\mathcal{M}_{\mathrm{pg}}(u, v) \boldsymbol{v} \cdot \nabla_{\boldsymbol{x}} n_{p}^{0}, \int_{\mathbb{R}^{2 N}} g_{p}^{0} \mathrm{~d} \boldsymbol{v} \mathrm{d} \boldsymbol{u}=0 .
$$

After some algebra, we find that the solution of this problem writes:

$$
g_{p}^{0}(t, \boldsymbol{x}, \boldsymbol{u}, \boldsymbol{v})=\left(\frac{\boldsymbol{u}}{S}+(2+S) \frac{\boldsymbol{v}}{S}\right) \cdot \nabla_{\boldsymbol{x}} n_{p}^{0}(t, \boldsymbol{x}) .
$$

The end of the proof is now straightforward.

\section{Conclusion}

In this paper ${ }^{1}$, we have proposed a hierarchy of simplified models for turbulent sprays, in the frame or forced homogeneous turbulence. The originality of the proposed analysis relies on the use of the joint particle-gas probability density function $f_{p g}$, which accounts for the particle velocity supplemented with the gas-fluctuating velocity along particle path.

The starting point of our approach is the Fokker-Planck type equation satisfied by $f_{p q}$, which models at the same time the motion of the particles and the turbulence seen by the particle, at time scales falling in the inertial range of the turbulence. When the system is observed at time scales much larger than either the dynamical response time of the particles, $\tau_{p}$, or the integral time scale of the turbulence, $\tau_{g}$, we have proved that it is possible to derive reduced models by using Chapman-Enskog type expansions. In this way, we recover classical results from Tchen, Hinze, Zaichik, and Reeks, concerning the modeling of particle diffusion by fluid turbulence. An important by-product of our proposed methodology is the determination of an explicit approximation formula for the joint probability function, in each asymptotic case considered in this paper.

\footnotetext{
${ }^{1}$ This document has been produced using $\mathrm{T}_{\mathrm{E}} \mathrm{X}_{\mathrm{MACS}}$ (see http://www.texmacs.org)
} 
We are presently investigating different generalizations of this work. On the one hand, in more realistic models, the drag force is a nonlinear function of the difference $\left(\boldsymbol{v}_{p}-\boldsymbol{U}_{g}\left(t, \boldsymbol{x}_{p}(t)\right)\right.$. It is only for small values of this "slipping" velocity that the the linear Stokes drag force is valid. Hence, considering such forces would provide nongaussian equilibrium distributions, especially for heavy particles. On the other hand, there are many models aimed at improving the description of the turbulent velocity $\boldsymbol{u}_{p}(t):=\boldsymbol{U}_{g}^{\prime}\left(t, \boldsymbol{x}_{p}(t)\right)$. See for example the work [16] and the references therein for a review. Here, one may consider two distinct cases.

The first case deals with flows in which the mean velocity of the career flow and the mean velocity of the particles are the same. This is a typical situation when the mean velocity of the gas is a constant, say zero. In that case, possibly after a transient, the mean velocity of the particles equals that of the gas, so that very heavy particles will mostly not move and therefore will "see" the eulerian properties of the turbulent flow, whereas the small particles will behave like fluid particles and therefore "see" the lagrangian properties of the turbulent flow. Estimating for a given size of particles which properties to consider is the so-called problem of the crossing-trajectory effect (see [23]).

The second case deals with flows where the mean velocity of the career flow and the mean velocity of the particles are significatively different, as is typical in gravitational settling of particles where the mean velocity of the carrier flow equals zero and the mean velocity of the particles equals the falling velocity. In that case, the autocorrelation time of the turbulence seen by the particles precisely depends on the ratio between the velocity of the particles and the eulerian spatial autocorrelation of the turbulent velocity. A similar difficulty arises when a particle moves in a nonuniform mean flow (see $[26,27,22]$ ).

We believe that the strategy and formalism adopted in the present paper can be of great help for tackling these difficulties. This is a work in progress.

Appendix A. Expression of the dissipation rate (see Eq.(5.4)).

For the sake of simplicity, we set $\mathcal{M}:=\mathcal{M}_{\mathrm{pg}}$ and $f:=f_{\mathrm{pg}}$. Using integration by parts, a straightforward calculation yields:

$$
\begin{aligned}
& \int_{\mathbb{R}^{2 N}} \mathcal{A}(f) \ln (f) \mathrm{d} \boldsymbol{v} \mathrm{d} \boldsymbol{u} \\
= & \int_{\mathbb{R}^{2 N}}\left[\frac{(\boldsymbol{v}-\boldsymbol{u})}{S} \cdot \nabla_{\boldsymbol{v}} f+\boldsymbol{u} \cdot \nabla_{\boldsymbol{u}} f+\bar{\sigma}_{g}^{2} \frac{\nabla_{\boldsymbol{u}} f \cdot \nabla_{\boldsymbol{u}} f}{f}\right] \mathrm{d} \boldsymbol{v} \mathrm{d} \boldsymbol{u} \\
= & \int_{\mathbb{R}^{2 N}}\left[-N \frac{1+S}{S} f+\bar{\sigma}_{g}^{2} \frac{\nabla_{\boldsymbol{u}} f \cdot \nabla_{\boldsymbol{u}} f}{f}\right] \mathrm{d} \boldsymbol{v} \mathrm{d} \boldsymbol{u} .
\end{aligned}
$$

We have easily:

$$
4 \mathcal{M} \nabla_{u} \sqrt{\frac{f}{\mathcal{M}}} \cdot \nabla_{u} \sqrt{\frac{f}{\mathcal{M}}}=\frac{\nabla_{\boldsymbol{u}} f \cdot \nabla_{\boldsymbol{u}} f}{f}+f \frac{\nabla_{\boldsymbol{u}} \mathcal{M} \cdot \nabla_{\boldsymbol{u}} \mathcal{M}}{\mathcal{M}^{2}}-2 \frac{\nabla_{\boldsymbol{u}} f \cdot \nabla_{\boldsymbol{u}} \mathcal{M}}{\mathcal{M}} .
$$

Hence, using the relation

$$
\nabla_{\boldsymbol{u}} \mathcal{M}=\frac{1+S}{S \bar{\sigma}_{g}^{2}}(\boldsymbol{v}-\boldsymbol{u}) \mathcal{M}
$$

we obtain

$$
4 \mathcal{M} \nabla_{u} \sqrt{\frac{f}{\mathcal{M}}} \cdot \nabla_{u} \sqrt{\frac{f}{\mathcal{M}}}=\frac{\nabla_{\boldsymbol{u}} f \cdot \nabla_{\boldsymbol{u}} f}{f}+\frac{(1+S)^{2}}{S^{2} \bar{\sigma}_{g}^{4}}|\boldsymbol{v}-\boldsymbol{u}|^{2}-2 \frac{1+S}{S \bar{\sigma}_{g}^{2}}(\boldsymbol{v}-\boldsymbol{u}) \cdot \nabla_{\boldsymbol{u}} f .
$$


Inserting this relation in (A.1), it finally follows that:

$$
\begin{aligned}
\int_{\mathbb{R}^{2 N}} \mathcal{A}(f) \ln (f) \mathrm{d} \boldsymbol{v} \mathrm{d} \boldsymbol{u}= & \int_{\mathbb{R}^{2 N}} 4 \mathcal{M}\left(\nabla_{u} \sqrt{\frac{f}{\mathcal{M}}} \cdot \nabla_{u} \sqrt{\frac{f}{\mathcal{M}}}\right) \mathrm{d} \boldsymbol{v} \mathrm{d} \boldsymbol{u} \\
& +\frac{1+S}{S}\left[N \int_{\mathbb{R}^{2 N}} f \mathrm{~d} \boldsymbol{v} \mathrm{d} \boldsymbol{u}-\int_{\mathbb{R}^{2 N}} \frac{1+S}{S} \frac{|\boldsymbol{v}-\boldsymbol{u}|^{2}}{\bar{\sigma}_{g}^{2}} f \mathrm{~d} \boldsymbol{v} \mathrm{d} \boldsymbol{u}\right] .
\end{aligned}
$$

\section{Appendix B. Proof of Proposition 3.3.}

First, we note that (3.1) yields:

$$
\mathcal{A}_{\boldsymbol{v}}\left(f_{p}\right)=K\left(\partial_{t} f_{p}+\operatorname{div}_{\boldsymbol{x}}\left(\boldsymbol{v} f_{p}\right)\right),
$$

where the operator $\mathcal{A}_{\boldsymbol{v}}$ is defined as:

$$
\mathcal{A}_{\boldsymbol{v}}(f)=\operatorname{div}_{\boldsymbol{v}}\left[-\boldsymbol{v} f-\frac{\bar{\sigma}_{g}^{2}}{S} \nabla_{\boldsymbol{v}} f\right] .
$$

Hence, when $K \rightarrow 0^{+}$, we formally get that $f_{p} \rightarrow f_{p}^{0}$ where $f_{p}^{0}$, satisfies the system:

$$
\left\{\begin{aligned}
\mathcal{A}_{\boldsymbol{v}}\left(f_{p}^{0}\right) & =0 \\
\int_{\mathbb{R}^{N}} f_{p}^{0} d \boldsymbol{v} & =n_{p}^{0} \\
(i i) \quad \partial_{t} n_{p}^{0}+\operatorname{div}_{\boldsymbol{x}}\left(\int_{\mathbb{R}^{N}} \boldsymbol{v} f_{p}^{0} d \boldsymbol{v}\right) & =0 .
\end{aligned}\right.
$$

It can easily be checked that the unique positive solution of $(i)$, under the constraint (ii), writes:

$$
f_{p}^{0}=\frac{S^{N / 2}}{\left(2 \pi \bar{\sigma}_{g}^{2}\right)^{N / 2}} \exp \left(\frac{-S \boldsymbol{v}^{2}}{2 \bar{\sigma}_{g}^{2}}\right) n_{p}^{0}
$$

and it results from (iii) that $n_{p}^{0}$ must solve:

$$
\partial_{t} n_{p}^{0}=0
$$

We must now compute the first order term in the series expansion of $f_{p}$ in powers of K. For all $K>0$, let us introduce the functions $n_{p}^{K}$ and $g_{p}^{K}$ such that by definition:

$$
n_{p}^{K}=\int_{\mathbb{R}^{N}} f_{p} \mathrm{~d} \boldsymbol{v}, \quad \int_{\mathbb{R}^{N}} g_{p}^{K} \mathrm{~d} \boldsymbol{v}=0,
$$

and

$$
f_{p}=\frac{S^{N / 2}}{\left(2 \pi \bar{\sigma}_{g}^{2}\right)^{N / 2}} \exp \left(\frac{-S \boldsymbol{v}^{2}}{2 \bar{\sigma}_{g}^{2}}\right) n_{p}^{K}+K g_{p}^{K}
$$

Equation (3.1) yields:

$$
\mathcal{A}_{\boldsymbol{v}}\left(g_{p}^{K}\right)=\frac{S^{N / 2}}{\left(2 \pi \bar{\sigma}_{g}^{2}\right)^{N / 2}} \exp \left(\frac{-S \boldsymbol{v}^{2}}{2 \bar{\sigma}_{g}^{2}}\right)\left(\partial_{t} n_{p}^{K}+\operatorname{div}_{\boldsymbol{x}}\left(\boldsymbol{v} n_{p}^{K}\right)\right)+K\left(\partial_{t} g_{p}^{K}+\operatorname{div}_{\boldsymbol{x}}\left(\boldsymbol{v} g_{p}^{K}\right)\right) .
$$


By construction, we know that $n_{p}^{K} \rightarrow n_{p}^{0}$ when $K \rightarrow 0^{+}$. Therefore, thanks to the fact that $n_{p}^{0}$ the ( B.1), it follows that, when $K \rightarrow 0^{+}, g_{p}^{K}$ tends formally towards $g_{p}^{0}$ solution of:

$$
\mathcal{A}_{\boldsymbol{v}}\left(g_{p}^{0}\right)=\frac{S^{N / 2}}{\left(2 \pi \bar{\sigma}_{g}^{2}\right)^{N / 2}} \exp \left(\frac{-S \boldsymbol{v}^{2}}{2 \bar{\sigma}_{g}^{2}}\right) \boldsymbol{v} \cdot \nabla_{x} n_{p}^{0}, \quad \int_{\mathbb{R}^{N}} g_{p}^{0} \mathrm{~d} \boldsymbol{v}=0 .
$$

The unique solution of this problem writes:

$$
g_{\mathrm{pg}}^{0}=-\frac{S^{N / 2}}{\left(2 \pi \bar{\sigma}_{g}^{2}\right)^{N / 2}} \exp \left(\frac{-S \boldsymbol{v}^{2}}{2 \bar{\sigma}_{g}^{2}}\right) \boldsymbol{v} \cdot \nabla_{x} n_{p}^{0}
$$

Since $n_{p}^{0}$ (respectively $g_{p}^{0}$ ), is the limit of $n_{p}^{K}$ (respectively $g_{p}^{K}$ ) when $K \rightarrow 0^{+}$, it follows that there exists (at least formally) a function $\varepsilon$ defined on $\left[0,+\infty\left[{ }^{2} \times \mathbb{R}^{2 N}\right.\right.$ such that $\forall t>0, \forall(\boldsymbol{x}, \boldsymbol{v}) \in \mathbb{R}^{2 N}, \lim _{K \rightarrow 0} \varepsilon(K, t, \boldsymbol{x}, \boldsymbol{v})=0$ and such that for all $K$ small enough:

$$
g_{p}^{K}=-\frac{S^{N / 2}}{\left(2 \pi \bar{\sigma}_{g}^{2}\right)^{N / 2}} \exp \left(\frac{-S \boldsymbol{v}^{2}}{2 \bar{\sigma}_{g}^{2}}\right) \boldsymbol{v} \cdot \nabla_{x} n_{p}^{K}+\varepsilon(K) .
$$

Inserting this last expression in ( B.2), we get:

$$
f_{p}=\frac{S^{N / 2}}{\left(2 \pi \bar{\sigma}_{g}^{2}\right)^{N / 2}} \exp \left(\frac{-S \boldsymbol{v}^{2}}{2 \bar{\sigma}_{g}^{2}}\right)\left(n_{p}^{K}-K \boldsymbol{v} \cdot \nabla_{x} n_{p}^{K}\right)+o(K) .
$$

A short calculation gives:

$$
\int_{\mathbb{R}^{N}} \boldsymbol{v} f_{p} d \boldsymbol{v}=-\frac{K \bar{\sigma}_{g}^{2}}{S} \nabla_{x} n_{p}^{K}+o(K) .
$$

Hence, using that $n_{p}^{K}=\int_{\mathbb{R}^{N}} f_{p} d \boldsymbol{v}$, we find that $n_{p}^{K}$ :

$$
\partial_{t} n_{p}^{K}+\operatorname{div}_{\boldsymbol{x}}\left(-\frac{K \bar{\sigma}_{g}^{2}}{S} \nabla_{\boldsymbol{x}} n_{p}^{K}\right)=o(K)
$$

finally, let $n_{p}$ be the solution of (3.7):

$$
\partial_{t} n_{p}-\operatorname{div}_{\boldsymbol{x}}\left(\frac{K \bar{\sigma}_{g}^{2}}{S} \nabla_{\boldsymbol{x}} n_{p}\right)=0
$$

Formally, it follows that $n_{p}=n_{p}^{K}+o(K)$ and $K \nabla n_{p}=K \nabla n_{p}^{K}+o(K)$. Hence, inserting these relations in (B.3), we finally obtain:

$$
f_{p}=\frac{S^{N / 2}}{\left(2 \pi \bar{\sigma}_{g}^{2}\right)^{N / 2}} \exp \left(\frac{-S \boldsymbol{v}^{2}}{2 \bar{\sigma}_{g}^{2}}\right)\left[n_{p}-K \boldsymbol{v} \cdot \nabla_{\boldsymbol{x}} n_{p}\right]+o(K)
$$

which is the result announced.

Acknowledgement. We would like to thank the second referee for his remarks and comments, which helped us improve the quality of the manuscript. 


\section{REFERENCES}

[1] N. Ben Abdallah and P. Degond, On a hierarchy of macroscopic models for semiconductors, J. Math. Phys., 37, 3306-3333, 1996.

[2] C. Cercignani, The Boltzmann Equation and its Applications, Appl. Math. Sci., SpringerVerlag, 67, 1988.

[3] C. Cercignani, R. Illner and M. Pulvirenti, The Mathematical Theory of Dilute Gases, SpringerVerlag, Berlin, 1994.

[4] J.-F. Clouet and K. Domelevo, Solution of a kinetic stochastic equation modeling a spray in a turbulent gas flow, Math. Models and Meth. in Appl. Sci., 7(2), 239-263, 1997.

[5] G. Csanady, Turbulent diffusion of heavy particles in the atmosphere, J. Atmos. Sci., 20, 201208, 1963.

[6] P. Degond, M. Lemou and M. Picasso, Voscoelastic fluid models derived from kinetic equations for polymers, SIAM J. Appl. Math., 62, 1501-1519, 2002.

[7] J.K. Duckowicz, A particle-fluid numerical model for liquid sprays, J. Comp. Physics, 35, 229253,1980 .

[8] T. Goudon, P.-E. Jabin and A. Vasseur, Hydrodynamic limit for the Vlasov-Navier-Stokes equations, II, fine particles regime, Indiana Univ. Math. J., 53(6), 1517-1536, 2004.

[9] T. Goudon and F. Poupaud, On the modeling of the transport of particles in turbulent flows, M2AN Math. Model. Numer. Anal., 38(4), 673-690, 2004.

[10] J.O. Hinze, Turbulence, McGraw-Hill Book Co., 1975.

[11] K. E. Hyland, S. Mckee and M. W. Reeks, Derivation of a pdf kinetic equation for the transport of particles in turbulent flows, J. Phys. A, 32(34), 6169-6190, 1999.

[12] P.-E. Jabin, Large time concentrations for solutions to kinetic equations with energy dissipation, Comm. Partial Differ. Equ., 25(3-4), 541-557, 2000.

[13] P.-E. Jabin, Macroscopic limit of Vlasov type equations with friction, Ann. Inst. H. Poincaré Anal. Non Linéaire, 17(5), 651-672, 2000.

[14] B. Lapeyre, E. Pardoux and R. Sentis, Mathématiques Applications, in Méthodes de MonteCarlo pour les Équations Transport et de Diffusion, Springer, 1998.

[15] J. Laviéville, E. Deutsch and O. Simonin, Large eddy simulation of interactions between colliding particles and a homogeneous isotropic turbulence field, Gas-Solid Flows, ASME, 228, 347-358, 1995.

[16] J.-P. Minier and E. Peirano, The pdf approach to turbulent polydispersed two-phase flows, Phys. Rep., 352(1-3), 1-214, 2001.

[17] J.-P. Minier and J. Pozorski, Analysis of existing lagrangian models and new propositions for particle dispersion in homogeneous stationary turbulence, Technical report, EDF, November 1992.

[18] J.-P. Minier and J. Pozorski, The pdf approach to turbulent polydispersed two-phase flows, Technical report, EDF, 1996.

[19] S.B. Pope, Consistency conditions for random-walk models of turbulent dispersion, Phys. Fluids, 30(8), 1987.

[20] S.B. Pope, Particle method for turbulent flows: Integration of stochastic model equations, J. Comput. Phys., 117, 332-349, 1995.

[21] S.B. Pope, Turbulent Flows, Cambridge University Press, 2000.

[22] S.B. Pope, Stochastic Lagrangian models of velocity in homogeneous turbulent shear flow, Phys. Fluids, 14(5), 1696-1702, 2002.

[23] J. Pozorski, J.-P. Minier and O. Simonin, Analysis and new propositions for the crossingtrajectory effect in lagrangian turbulent dispersion models, ASME, FED, Gas-Solid Flows, $166,1993$.

[24] M.W. Reeks, On the dispersion of small particles suspended in an isotropic turbulent fluid, J. Fluid Mech., 83(3), 529-546, 1977.

[25] M.W. Reeks, On a kinetic equation for the transport of particles in turbulent flows, Phys. Fluids A, 3(3), 446-456, 1991.

[26] M.W. Reeks, On the constitutive relations for the dispersed particles in nonuniform flows, i: Dispersion in a simple shear, Phys. Fluids A, 5(3), 750-761, 1993.

[27] M.W. Reeks, On pdf equations and their solution for particle dispersion in a simple shear, in 5th International Conference on Multiphase Flow, ICMF'04, Yokohama, Japan, May 30-June 4, 2004.

[28] O. Simonin, E. Deutsch and J.-P. Minier, Eulerian prediction of the particle/fluid correlated motion in turbulent two-phase flows, Appl. Scientific Research, 51, 275-283, 1993.

[29] G.I. Taylor, The conditions necessary for discontinuous motion in gases, Proc. London Math. Soc., 2, 196-212, 1921. 
[30] C.M. Tchen, Mean value and correlation problems connected with the motion of small particles suspended in a turbulent fluid, PhD thesis, Delft, The Hague, Martinus Nijhoff, 1947.

[31] M.I. Yudine, Physical considerations on heavy particle diffusion, Advances in Geophysics, 6, 185-191, 1959.

[32] L.I. Zaichik, O. Simonin and V.M. Alipchenkov, Two statistical models for predicting collision rates of inertial particles in homogeneous isotropic turbulence, Phys. Fluids, 15(10), 29953005, 2003.

[33] L.I. Zaichik, An equation for the particle velocity probability density function in inhomogeneous turbulent flow, Fluid Dynamics, 32(2), 261-267, 1996.

[34] L.I. Zaichik, Modeling the motion of particles in a nonuniform turbulent flow on the basis of the equation for the probability density function, Prikl. Mat. Mekh., 61(1), 132-138, 1997. 